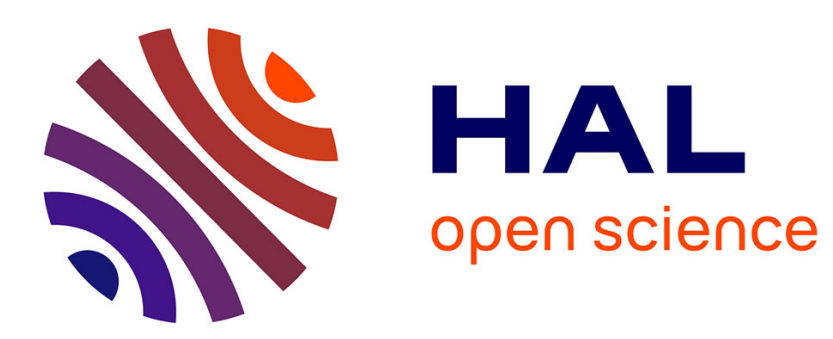

\title{
Analyse de la présidence de Ma Ying-jeou à mi-mandat. De la difficulté à livrer la (bonne) marchandise
}

\author{
Frank Muyard
}

\section{To cite this version:}

Frank Muyard. Analyse de la présidence de Ma Ying-jeou à mi-mandat. De la difficulté à livrer la (bonne) marchandise. Perspectives chinoises, 2010, 112, pp.5 - 23. 10.3406/perch.2010.4004 . halshs-02515505

\author{
HAL Id: halshs-02515505 \\ https://shs.hal.science/halshs-02515505
}

Submitted on 23 Mar 2020

HAL is a multi-disciplinary open access archive for the deposit and dissemination of scientific research documents, whether they are published or not. The documents may come from teaching and research institutions in France or abroad, or from public or private research centers.
L'archive ouverte pluridisciplinaire HAL, est destinée au dépôt et à la diffusion de documents scientifiques de niveau recherche, publiés ou non, émanant des établissements d'enseignement et de recherche français ou étrangers, des laboratoires publics ou privés. 


\section{Analyse de la présidence de Ma Ying-jeou à mi-mandat. De la difficulté à livrer la (bonne) marchandise}

Frank Muyard

\section{Résumé}

Depuis son élection comme président de Taiwan en 2008, Ma Ying-jeou s'est engagé dans une politique active de rapprochement avec la Chine, conduisant à la signature d'une série d'accords économiques et commerciaux qui ont normalisé et libéralisé les relations économiques inter-détroit. Mais la manière avec laquelle ce rapprochement est mené, combinée à la crise économique qui a frappé Taiwan pendant l'essentiel des deux premières années du mandat de Ma, et une accumulation de faux pas et de gestion déficiente par le président et le gouvernement Kuomintang (KMT) ont engendré une crise de confiance et un mécontentement massif au sein de la population taiwanaise. Cela se reflète dans des taux de popularité continuellement bas et une série de revers électoraux en 2009 et 2010, ainsi que dans la résurgence d'une opposition reformée sous la direction de Tsai Ing-wen. La divergence entre le nationalisme chinois de plus en plus apparent de Ma et l'identité nationale taiwanaise majoritaire est une indication supplémentaire de la déconnexion existant entre le gouvernement KMT et la majorité de la population.

\section{Citer ce document / Cite this document :}

Muyard Frank. Analyse de la présidence de Ma Ying-jeou à mi-mandat. De la difficulté à livrer la (bonne) marchandise. In: Perspectives chinoises, n¹12, 2010. pp. 5-23;

doi : https://doi.org/10.3406/perch.2010.4004

https://www.persee.fr/doc/perch_1021-9013_2010_num_112_3_4004

Fichier pdf généré le 29/03/2019 


\section{Analyse de la présidence de}

Ma Ying-jeou à mi-mandat

De la difficulté à livrer la (bonne) marchandise

FRANK MUYARD

Depuis son élection comme président de Taiwan en 2008, Ma Ying-jeou s'est engagé dans une politique active de rapprochement avec la Chine, conduisant à la signature d'une série d'accords économiques et commerciaux qui ont normalisé et libéralisé les relations économiques inter-détroit. Mais la manière avec laquelle ce rapprochement est mené, combinée à la crise économique qui a frappé Taiwan pendant l'essentiel des deux premières années du mandat de Ma, et une accumulation de faux pas et de gestion déficiente par le président et le gouvernement Kuomintang (KMT) ont engendré une crise de confiance et un mécontentement massif au sein de la population taiwanaise. Cela se reflète dans des taux de popularité continuellement bas et une série de revers électoraux en 2009 et 2010, ainsi que dans la résurgence d'une opposition reformée sous la direction de Tsai Ing-wen. La divergence entre le nationalisme chinois de plus en plus apparent de Ma et l'identité nationale taiwanaise majoritaire est une indication supplémentaire de la déconnexion existant entre le gouvernement KMT et la majorité de la population.

$\mathrm{D}$ eux ans après les larges victoires de Ma Ying-jeou et du Kuomintang (KMT) aux élections présidentielles et législatives taiwanaises, le nouveau gouvernement du KMT et son président font face à un fort mécontentement de la population taiwanaise. Même si la lune de miel de Ma avec l'électorat promettait d'être courte compte tenu des multiples défis économiques et politiques auxquels Taiwan était confronté en 2008, la chute spectaculaire de sa cote de popularité a surpris de nombreux observateurs ${ }^{(1)}$. La politique de rapprochement avec la Chine de Ma Ying-jeou a produit des résultats significatifs et suscité soutien et applaudissements de la plupart des capitales étrangères. Mais la façon dont cette politique a été menée et une série de faux pas et de manquements du gouvernement et de Ma luimême se sont alliés à la crise économique mondiale pour produire une grave crise de confiance au sein de l'opinion concernant leurs compétences administratives ainsi que leur réel attachement aux intérêts et à la souveraineté de Taiwan par rapport à la Chine. Cet article se penche sur les deux premières années du gouvernement de Ma Ying-jeou et ses politiques, leur niveau de succès comme les résistances qu'elles suscitent, et en particulier sur les principaux défis auxquels il a été confronté : les relations avec la Chine, l'économie, la politique intérieure et les élections régionales ainsi que la question de l'identité nationale.

\section{Politiques et résultats de Ma Ying-jeou et du KMT depuis 2008}

En 2008, les élections présidentielles et législatives ont donné au KMT une mainmise écrasante sur toutes les institutions politiques nationales. Au parlement, le KMT et ses alliés du camp bleu (le PFP et le NP) ont remporté environ $55 \%$ des voix et plus des deux tiers des sièges du nouveau Yuan législatif (YL) composé de 113 sièges, renforçant leur majorité et le contrôle qu'ils exercent sur le pouvoir législatif taiwanais depuis la fin des années 1940. Lors de l'élection présidentielle, le tandem du Kuomintang composé de Ma Ying-jeou et de son vice-président Vincent Siew (Hsiao Wan-chang) est sorti largement vainqueur avec 58,5\% des voix contre 41,5\% pour leurs adversaires du Parti démocrate progressiste (Minjindang, ci-après DPP selon l'acronyme anglais) Frank Hsieh Chang-ting et Su Tseng-chang ${ }^{(2)}$. Surfant sur la vague d'insatisfaction contre le gouvernement $d u$ DPP et le président sortant Chen Shui-bian impliqués dans des scandales de corruption et critiqués pour leur mauvaise

1. Shelley Rigger, « Ma’s Puzzling Midterm Malaise », Brookings Northeast Asia Commentary, $n^{\circ} 37,11$ mars 2010.

2. Cf. Frank Muyard, «Élections 2008 à Taiwan. Victoire de Ma Ying-jeou et retour du KMT aux affaires ", Perspectives chinoises, $n^{\circ} 102,2008 / 1$, p. 82-98. 
gestion de l'économie du pays, des affaires administratives et des relations extérieures, en particulier avec la Chine et les États-Unis, Ma Ying-jeou a mené une campagne fondée sur trois piliers : la relance de l'économie, le rapprochement avec la Chine et la lutte contre la corruption, ainsi que sur un engagement fort à protéger la souveraineté et l'identité de Taiwan. Son programme économique "6-3-3 " promettait 6 $\%$ de croissance annuelle du PIB, moins de 3 \% de chômage et, en 2016, un PIB par habitant de 30000 dollars. Ma défendait également le développement de relations économiques plus étroites avec la République populaire de Chine $(\mathrm{RPC})$, des relations présentées en termes pragmatiques comme le meilleur moyen de renforcer l'économie taiwanaise et la stabilité de la région, en commençant par l'établissement des trois liaisons directes (aériennes, maritimes et postales) entre les deux pays ainsi que par l'ouverture de Taiwan aux touristes et investissements chinois. Sur l'aspect des relations politiques avec la Chine, Ma avait annoncé une politique " de non-unification, de non-indépendance, et de non-usage de la force ", en s'engageant à ne pas entreprendre de négociations concernant l'unification pendant son mandat. En matière de politique intérieure, Ma promettait un gouvernement hautement efficace et compétent en contraste avec les allégations d'incompétence et de corruption visant le DPP. Enfin, il appelait à une politique de réconciliation nationale après huit ans de polarisation partisane.

\section{Les relations entre Taiwan et la Chine}

Le rapprochement entre Taiwan et la Chine est au cœur de la politique nationale du KMT depuis son retour au pouvoir et c'est dans ce domaine que les résultats les plus concrets peuvent être constatés. Trois aspects de cette politique seront analysés ici : les accords inter-détroit, la question de la souveraineté de Taiwan et l'espace diplomatique de Taiwan. Bien que des progrès dans les relations inter-détroit étaient attendus avec l'arrivée de la nouvelle administration au pouvoir, le gouvernement de $\mathrm{Ma}$ en a surpris beaucoup par la rapidité et la priorité donnée aux relations avec Pékin plutôt que de se concentrer sur la relance de l'économie tel que cela avait été annoncé lors de la campagne présidentielle. La raison en est que le KMT perçoit le développement économique futur de Taiwan comme dépendant de son intégration à la sphère économique chinoise et que son objectif d'unification finale exige au préalable une intensification des relations économiques et culturelles avec la Chine. La nouvelle politique chinoise de Ma et du KMT prône par conséquent un rapprochement économique, symbolique et politique progressif avec la Chine avec quatre objectifs : la baisse de la tension politique et militaire dans le détroit de Formose, le renforcement des liens économiques avec la Chine afin d'intégrer étroitement les deux économies et créer in fine une forme de marché commun, la lutte contre le mouvement indépendantiste taiwanais, et la préparation de conditions favorables à une possible unification dans un avenir encore indéterminé.

En pratique, l'investiture de Ma à la présidence de la République de Chine (RDC) le 20 mai 2008 a été rapidement suivie par la reprise des pourparlers officiels avec la RPC à travers les canaux établis des institutions semi-gouvernementales que sont la Fondation pour les échanges dans le détroit (SEF) pour Taiwan et l'Association pour les relations à travers le détroit de Taiwan (ARATS) pour la Chine. Leur première rencontre en dix ans s'est tenue à Pékin en juin 2008 et a officialisé la finalisation des accords sur les vols charters directs le week-end et l'ouverture de Taiwan aux touristes chinois venant directement de Chine. Ces accords avaient été largement négociés et conclus en 2007 par le précédent gouvernement de Chen Shui-bian mais avaient été retardés par Pékin afin d'attendre le retour du KMT au pouvoir. Depuis, "les rencontres Chiang-Chen " entre la SEF et l'ARATS ont eu lieu tous les six mois ${ }^{(3)}$ et ont abouti, à la fin 2009, à la signature d'un total de 12 accords techniques entre les deux parties concernant les vols réguliers directs (passagers et marchandises), les liaisons maritimes, les services postaux, les touristes chinois, la sécurité alimentaire, la lutte contre la criminalité et l'assistance juridique mutuelle, les normes et la métrologie, l'inspection et la quarantaine des produits agricoles, les équipages de pêche et la coopération financière, ainsi qu'à trois protocoles d'accord financiers distincts ${ }^{(4)}$. Taipei a également adopté une série de mesures unilatérales afin de faciliter les relations économiques avec la Chine. Il a élargi l'accès aux mini-liaisons directes (par les îles Kinmen et Matsu), autorisé l'échange à Taiwan de la monnaie de la RPC, le renminbi, augmenté le montant maximum des investissements taiwanais en Chine, autorisé les maires et les chefs de comtés taiwanais à voyager en Chine, limité les restrictions concernant les investissements taiwanais dans des fonds étrangers ayant

3. L'appellation Chiang-Chen fait référence aux noms des deux principaux négociateurs : le président de la SEF, Chiang Pin-kun, et le président de l'ARATS, Chen Yunlin.

4. Pour plus de détails sur ces accords inter-détroit et leur négociation ainsi que sur la politique actuelle du gouvernement de Ma envers la Chine continentale, voir les sites du Conseil des Affaires continentales de Taiwan (MAC), (http://www.mac.gov.tw/ ct.asp?xltem $=67145 \& C+$ Node $=5710 \& \mathrm{mp}=1$ ), et de la SEF (http://www.sef.org.tw/lp. asp?ctNode $=4382 \&$ CtUnit $=2567 \&$ BaseDSD $=21 \& \mathrm{mp}=300$ ). 
des actifs chinois, simplifié le processus d'accueil des experts professionnels chinois à Taiwan, et assoupli les règles concernant les mises en bourse d'entreprises étrangères et les investissements de fonds chinois à la Bourse de Taiwan. Par conséquent, les portes vers la Chine sont maintenant ouvertes aux investissements de la plupart des secteurs de haute technologie taiwanais ainsi qu'aux secteurs bancaire, financier et de l'assurance, avec toutefois certaines limites concernant la quantité du capital et des ressources et les niveaux de technologie transférés dans les opérations chinoises afin de garantir le maintien par ces entreprises d'une base solide à Taiwan. Les investissements chinois sont par ailleurs désormais autorisés, et c'est une première, dans 100 secteurs de l'économie ${ }^{(5)}$. En mai 2010, Taipei a ouvert un Bureau du tourisme à Pékin, la RPC inaugurant à la suite un bureau similaire à Taipei, constituant ainsi les premières institutions quasi officielles de chaque partie sur le territoire de l'autre ${ }^{(6)}$. D'intenses négociations ont également eu lieu à propos d'un accord économique et de libre-échange inter-détroit portant le nom d'ECFA (Economic Cooperation Framework Agreement), finalement signé à la fin juin 2010 (voir ci-dessous).

Tous ces accords sont le signe d'une amélioration très nette des relations entre Taiwan et la Chine, pas seulement sur le plan économique mais aussi sur le plan politique. Cependant, les bénéfices concrets de ces interactions rapprochées ne sont pas toujours évidents pour la majorité de la population, principalement préoccupée par la reprise économique après deux ans de stagnation et de récession. De plus, la politique chinoise de Ma suscite l'opposition déterminée du camp vert mené par le DPP qui critique le cadre d'« une Chine unique " adopté par Ma et les soubassements politiques des pourparlers inter-détroit actuels, et dénonce les risques d'une dépendance économique excessive envers la Chine. Le manque de transparence avec lequel la politique de rapprochement de Ma est menée et son ambiguité quant à la nature de l'État taiwanais, constituent également de grands motifs d'inquiétude pour les Taiwanais.

La conclusion des récents accords inter-détroit a en effet été rendue possible par l'acceptation mutuelle du soi-disant " consensus de 1992 " (7), l'adhésion de Ma au principe d' " une Chine unique ", ou une de ses versions, et son rejet déclaré de l'indépendance formelle de Taiwan en tant qu'État séparé. Tout en rejetant toute revendication de souveraineté de la RPC sur Taiwan, Ma Ying-jeou et le KMT soutiennent que Taiwan est la République de Chine, un État chinois indépendant et souverain depuis sa fondation en 1912, et qu'en tant que tel il n'a pas besoin de réaffirmer son indépendance. L'ambiguité de ces affirmations est au cœur du compromis entre les deux parties leur permettant d'entamer des discussions. Elle assure que chacune mène officiellement une "politique d'une Chine unique " et rejette les idées de "deux Chines " ou de " une Chine, un Taiwan " qui étaient défendues sous une forme ou une autre par les anciens présidents Lee Teng-hui et Chen Shui-bian et qui reflètent plus fidèlement la réalité sur le terrain.

Cette absence de clarté sur le statut de Taiwan, bien qu'utile pour traiter avec la Chine, génère une forte opposition à Taiwan, particulièrement après une série de déclarations de $\mathrm{Ma}$ suggérant un nationalisme chinois plus profond qu'exprimé durant sa campagne présidentielle. L'assertion par Ma d'une forte identité taiwanaise et sa défense de la souveraineté et de la dignité de Taiwan avant les élections de 2008 ont fait beaucoup pour apaiser les craintes des Taiwanais concernant son sentiment d'appartenance nationale, et ont permis la première élection démocratique d'un Continental (Waishengren) à la présidence de Taiwan. Une fois au pouvoir, Ma et ses ministres ont toutefois continuellement mis l'accent sur la République de Chine et sur l'appartenance des Taiwanais à la nation ou à l'ethnicité chinoise (Zhonghua minzu) aux dépens de Taiwan et de l'identité taiwanaise, non seulement en faisant référence au nom du pays mais aussi à son " authentique " identité nationale ${ }^{(8)}$. Contrairement au DPP et

5. Ting-I Tsai, « In Big Shift, Taiwan Allows Investment From China », Wall Street Journal, 1er juillet 2009.

6. «Taiwan Opens Tourism Office in Beijing», United Daily News, 4 mai 2010.

7. Une expression inventée par Su Chi, l'ancien ministre du Conseil des Affaires continentales (MAC) de Taiwan, en février 2000, qui prétend que dans les discussions entre la SEF et l'ARATS en 1992 à Singapour, les deux parties se sont mises d'accord pour reconnaître qu'il y a " une seule Chine avec différentes interprétations » (yi zhong ge biao) ce qui mena à la signature d'une première série d'accords techniques entre Taiwan et la Chine. Cependant, ce « consensus de 1992 » n'a jamais été reconnu ni par le gouvernement de Lee Teng-hui, ni par la RPC dans les années 1990. Sur la base de documents officiels de négociation et du conseil de Lee Teng-hui, le gouvernement de Chen Shui-bian rejeta l'invention de Su Chi. Après sa victoire électorale, Ma Ying-jeou annonça que son gouvernement la reconnaîtrait en même temps que le principe d' " une Chine unique " avec la réserve des différentes interprétations, signifiant que pour Taipei l'expression « une seule Chine » fait référence à la République de Chine (RDC). La RPC ne reconnaît pas cette lecture et maintient que les deux parties se sont mises d'accord sur le principe d' " une Chine unique » en 1992, et le pose en préalable de tout pourparler avec Taiwan. Su Chi a été le chef du Conseil de sécurité nationale de Ma de mai 2008 à février 2010.

8. Terme polysémique, minzu peut signifier nation, nationalité, groupe ethnique, ou groupe national sans prétention reconnue au statut d'État-nation (comme dans shaoshu minzu, les « minorités nationales » de la RPC). À Taiwan, minzu a une forte connotation ethnoculturelle. Pour traduire l'idée de nation dans le sens d'« État-nation », minzu a été remplacé dans les travaux universitaires contemporains par le néologisme guozu. Groupe ethnique est rendu par zuqun. Ethnologie est traduit par minzuxue. L'usage de Zhonghua minzu par Ma Ying-jeou reflète les fondements de son nationalisme chinois, selon lesquels la nation est vue en termes essentialistes et culturalistes et se construit sur la filiation du sang et les origines ethniques. Pour Ma, les deux côtés du détroit de Taiwan appartiennent à une seule nation ethnique chinoise (qui, comme Ma l'a rhétoriquement dit à plusieurs reprises, descend du mythique Empereur Jaune) au-delà de leurs différences de citoyenneté, d'histoires et de trajectoires politiques. Ceci contraste fortement avec la façon dont les Taiwanais se perçoivent eux-mêmes aujourd'hui (voir la dernière partie de l'article). 
au KMT de Lee Teng-hui qui déclaraient que Taiwan est la RDC et la RDC est Taiwan, Ma Ying-jeou adhère seulement au premier énoncé et lui donne une tournure non sans conséquences : Taiwan est la RDC aujourd'hui, mais seulement une partie, car la RDC n'est pas limitée à Taiwan mais inclut également la Chine continentale. Miroir de la définition de la RPC par Pékin et de sa revendication de souveraineté territoriale sur Taiwan, cet énoncé s'éloigne toutefois radicalement du consensus formé progressivement à Taiwan depuis 1991 lorsque le gouvernement de Lee Teng-hui abandonna toute revendication sur la Chine continentale. Dans une interview donnée au quotidien mexicain $E l$ Sol en septembre 2008, Ma déclarait alors que Taiwan comme la Chine continentale sont constitutionnellement des " régions " de la RDC, une position réitérée depuis par le bureau présidentiel. Par conséquent, pour Ma Ying-jeou, les relations entre Taiwan et la Chine ne sont pas des relations d'État à État comme pour Chen Shui-bian (un pays de chaque côté du détroit, yi bian yi guo), ni des « relations spéciales d'État à État " (teshu guo yu guo guanxi) comme pour Lee Teng-hui, mais « des relations spéciales de région à région sur un pied d'égalité " (duideng diqu dui diqu teshu de guanxi) dans le cadre de la constitution de la RDC ${ }^{\left({ }^{(9)}\right.}$. Cette rétrogradation de Taiwan au simple statut de "région " plutôt que de pays indépendant portant le nom de RDC, limite Taiwan à un cadre pan-chinois, et non seulement nie le droit à l'auto-détermination des Taiwanais mais réduit aussi leur identité et leur sentiment de développement historique spécifique à une identité provinciale et à un particularisme au sein d'une nation chinoise élargie. Aggravées par l'incapacité de Ma Ying-jeou à obtenir que Chen Yunlin s'adresse à lui en tant que président de Taiwan lors de sa visite à Taipei pour la deuxième rencontre Chiang-Chen en décembre 2008, ces déclarations ont généré un large ressentiment au sein de la population Taiwanaise, y compris au sein du camp bleu, à l'égard de Ma, de sa politique chinoise et de son incapacité à défendre la dignité nationale de Taiwan. L'affirmation croissante par Ma Ying-jeou et son gouvernement de leur nationalisme chinois, qui s'inscrit dans la lignée de la vieille garde du KMT rassemblée autour de Lien Chan, Wu Po-Hsiung et de la plupart des politiciens continentaux de la deuxième génération, annonce un retour à l'idéologie sinocentrée du KMT pré-Lee Teng-hui, oblitérant deux décennies de démocratisation et de taiwanisation.

Cependant, jusqu'à présent la sensibilité entourant la question du statut politique de Taiwan a conduit Ma à rejeter tout pourparler politique avec la Chine ${ }^{(10)}$. Insatisfait du manque de clarté et de progrès à ce sujet, Pékin a tenté de faire pression sur Ma à l'automne 2009, mais a dû reculer devant les énormes réticences de Taipei ${ }^{(I I)}$. Non seulement n'y a-t-il pas de consensus à Taiwan autour de ces pourparlers, mais ceux-ci pourraient facilement devenir un champ de mines pour le KMT déjà suspecté d'abandonner la souveraineté de Taiwan dans les accords inter-détroit avec la Chine et sur la scène internationale. Quelques timides ouvertures concernant un accord de paix et des mesures de construction de la confiance (CBM) sur le plan militaire ont été tentées, mais sont aujourd'hui repoussées à plus tard. Tout progrès devrait toutefois satisfaire la promesse de Ma de ne pas entamer des pourparlers de paix avant un retrait préalable des missiles de la RPC dirigés vers Taiwan ${ }^{(12)}$.

Sur le front international, de légers progrès ont été faits. Appendice de l'entente autour du principe $d$ ' " une Chine unique " avec Pékin, Ma a proposé une " trêve diplomatique " et une politique de " non-négation mutuelle ", signifiant que chaque côté devrait éviter de nier l'existence de l'autre et s'abstenir de débaucher les alliés diplomatiques de l'autre ${ }^{(13)}$. Taiwan a renoncé publiquement à toute forme de diplomatie du chéquier, qui, dans une intense compétition avec Pékin, perdurait depuis deux décennies, chacun offrant aides au développement et autres avantages à des pays étrangers afin de maintenir ou de gagner leur reconnaissance diplomatique. Rompant avec la pratique des gouvernements précédents depuis 1993, l'administration de Ma s'est également abstenue ces deux dernières années de déposer une demande officielle d'adhésion de Taiwan à l'ONU, justifiant cette nouvelle stratégie du profil bas par l'échec des tentatives précédentes ${ }^{(14)}$. Le gouvernement n'a toutefois dévoilé aucune nouvelle politique claire concernant la position internationale de Taiwan autre que l'obtention graduelle du statut d'observateur dans des organisations techniques sous l'égide de l'ONU.

9. Cf. Ko Shu-ling, « "State To State" Theory is Dead, Ma Says », Taipei Times, 4 septembre 2008, et Ko Shu-ling, « Ma Refers to China as ROC Territory in Magazine Interview », Taipei Times, 8 octobre 2008.

10. Howard Schneider, "Taiwan's Ma Sets Limits on China Policy ", Washington Post, 7 ma 2010.

11. "No Consensus on China and Taiwan's "1992 Consensus" ", Central News Agency, 14 novembre 2009 ; " PRC Draws Bottom Line on Taiwan for KMT », Taiwan News, 17 novembre 2009. Voir aussi Alan Romberg, "The Winter of PRC Discontent », China Leadership Monitor, n 31, hiver 2010.

12. Cf. "Time Not Ripe for Cross-strait Military CBM Talks », United Daily News, 18 mars 2010 ; «Taiwan to China: Remove Missiles Before Peace Talks », Reuters, 6 avril 2010. Sur les aspects militaires et stratégiques des relations du gouvernement Ma avec la Chine, cf. Jean-Pierre Cabestan, "La nouvelle détente dans le détroit de Taiwan : quel impact sur la sécurité et l'avenir de la République de Chine ? » dans ce numéro.

13. “"Mutual Non-Denial” Remains, Ma Says », Taipei Times, 6 avril 2008.

14. " No Bid for Full UN Membership », Taipei Times, 16 août 2008; « No New UN Bid this Year: MOFA », Taipei Times, 13 août 2010. 
Non reconnue officiellement, la trêve semble néanmoins être en pratique respectée par la RPC. Depuis 2008, Taiwan n'a perdu aucun de ses alliés diplomatiques (23 en tout, situés principalement en Amérique Centrale et du Sud, en Afrique et dans le Pacifique) bien que les gouvernements nouvellement élus au Paraguay, au Nicaragua et au Salvador avaient précédemment fait connaitre leur souhait de transférer leur reconnaissance à la Chine. En mai 2009, et de nouveau en 2010, Taiwan s'est aussi vu accordé le statut d'observateur à l'AMS (l'Assemblée annuelle de l'Organisation mondiale de la santé ou OMS), un objectif poursuivi depuis longtemps par les gouvernements successifs à Taipei et une première occasion depuis 1971 de contacts internationaux officiels, même si limités, de Taiwan dans un forum lié à I'ONU ${ }^{(15)}$. Le choix de l'ancien vice-président et Premier ministre Lien Chan pour représenter Taiwan aux congrès des chefs d'État 2008 et 2009 de la Coopération économique pour l'Asie-Pacifique (APEC) au Pérou et à Singapour a été saluée dans certains cercles comme un progrès pour Taipei, toujours empêché par Pékin d'y envoyer son président ou son ministre des Affaires étrangères. Le fait que Pékin favorisait la nomination de Lien Chan, un des hommes politiques du KMT les plus prochinois et l'initiateur du programme politique commun KMT-PCC de 2005 contre l'indépendance de Taiwan, n'a cependant rien fait pour dissiper l'impression du camp vert que les efforts de Ma pour élargir l'espace diplomatique de Taiwan restent sous le ferme contrôle de Pékin. En effet, mis à part le cas de l'AMS, qui était considéré par Taipei et les pays occidentaux comme un test minimum de la bonne volonté de la $\mathrm{RPC}$ à répondre aux ouvertures et aux concessions de $\mathrm{Ma}$, Pékin n'a fait preuve d'aucune flexibilité ni n'a concédé aucun espace, en paroles ou en actes, quant à la participation de Taiwan aux institutions internationales, y compris de mineures comme l'Organisation de l'aviation civile internationale (OACI) ou la Convention-cadre des Nations unies sur le changement climatique (CCNUCC). Si cette situation ne s'améliore pas et que la Chine ne répond pas de manière plus significative aux demandes de la population taiwanaise pour une meilleure représentation internationale de l'île, tant $\mathrm{Ma}$ que la Chine risquent de perdre toute confiance des Taiwanais envers cette politique de retenue. Alors que la majorité des Taiwanais est favorable à la détente dans le détroit et au maintien du statu quo actuel, les sondages montrent qu'ils exigent aussi continuellement le respect de leur souveraineté et de leur indépendance, et désirent ardemment une reconnaissance et un statut internationaux.
Dans l'ensemble, la détente politique entre les deux rives du détroit ainsi que la signature d'une série d'accords techniques avec Pékin facilitant les échanges commerciaux et les transports entre Taiwan et la Chine sont des avancées importantes pour le gouvernement de Ma et, en tant que telles, ont reçu un large soutien de la plupart des capitales étrangères. Leur coût possible en termes de souveraineté et d'autonomie de Taiwan, évident pour l'opposition taiwanaise, n'est pas bien perçu à l'étranger où la principale préoccupation est de maintenir le calme et la stabilité dans le détroit, et où la réalité et la profondeur du sentiment d'identité nationale particulier de Taiwan est toujours loin d'être largement compris. Une certaine inquiétude concernant un alignement trop étroit de Taipei sur Pékin s'est toutefois exprimé à Tokyo et à Washington, le Japon étant particulièrement choqué par la position fortement antijaponaise exprimée par le gouvernement de Ma à la suite d'un incident près des îles Diaoyutai (Senkaku en japonais) juste après son arrivée aux affaires ${ }^{(16)}$. Depuis, Taipei s'est efforcé de réassurer ses alliés traditionnels que le nouveau tropisme chinois de Ma ne se ferait pas aux dépens de leurs intérêts dans la région. Taiwan a récemment signé un vaste protocole d'accord pour renforcer la coopération et les échanges avec Tokyo afin de relancer sur de meilleures bases les relations traditionnellement proches entre les deux pays et leurs peuples ${ }^{(17)}$. L'administration Obama a, pour sa part, soutenu la politique de Ma et lui a permis de transiter plusieurs fois par les États-Unis lors de ses voyages chez les alliés diplomatiques de Taiwan dans l'hémisphère occidental. Certaines frictions sont cependant apparues à la suite du fiasco provoqué par les faux-pas du gouvernement de Ma concernant la ré-autorisation de la vente de bœuf américain à Taiwan, et certaines rencontres importantes pour faire avancer les négociations du TIFA ont été annulées ${ }^{(18)}$.

Il est de la plus haute importance pour Taiwan de conserver le soutien de Washington étant donné que le seul domaine dans lequel aucun progrès n'a été fait dans les relations inter-

15. Le nom et le statut de Taiwan à l'AMS semblent cependant avoir suivi les injonctions chinoises d'inclure le pays en tant que partie de la RPC, et provoque de ce fait la forte opposition du DPP qui y voit un exemple de la perte de souveraineté et de dignité nationale impliquée par la politique diplomatique de Ma. Cf. Vincent Y. Chao, « Former Official Laments Nation's Title at WHA », Taipei Times, 6 mai 2010.

16. " Taiwan Recalls its Japan Envoy over Diaoyutai Islands Clash », China Post, 15 juin 2008; I-chung Lai, "Taiwan and the Changing Strategic Balance in the East China Sea ", China Brief, vol. 9, $n^{\circ}$ 24, 3 décembre 2009.

17. Martin Williams, « Taiwan, Japan Sign Memorandum to Enhance Ties », Kyodo, 30 avril 2010.

18. Cf. Ting-I Tsai, «Taiwan Leader Vows to Repair U.S. Ties », Wall Street Journal, 6 janvier 2010 ; Alan Romberg, "Weathering the Storm », China Leadership Monitor, n 30, automne 2009. 
détroit est la sphère militaire. Pékin a non seulement rejeté la demande de Ma de retirer ses missiles pointés sur Taiwan, mais a également continué à renforcer leur nombre et leur capacité ainsi que ses capacités militaires générales dirigées vers l'île et destinées à dissuader tout assistance militaire américaine à Taiwan dans l'éventualité d'un conflit armé dans le détroit ${ }^{(19)}$. L'autorisation récente de ventes d'armes à Taiwan par l'administration Obama a également provoqué la condamnation la plus virulente de Pékin de ces dernières années, condamnation agrémentée d'un appel au boycott des entreprises américaines et de menaces de représailles (le tout dirigé vers Washington sans aucune critique adressée à Taipei). Le contrat d'armement américains avait pourtant déjà été annoncé par la précédente administration Bush et il est loin de pouvoir rétablir l'équilibre militaire dans le détroit. Comme les mois suivants l'ont montré, Pékin est apparemment allé trop loin sur cette affaire. S'ajoutant à d'autres actions et déclarations hostiles et inopportunes dans différents domaines d'interaction avec l'Occident pendant l'hiver 2009-2010, elle a suscité des réactions anti-Pékin et une crainte grandissante au sein des démocraties occidentales que la montée en puissance de la Chine ne se fasse pas aussi pacifiquement qu'elle le prétend ${ }^{(20)}$. Pendant ce temps, l'équilibre militaire dans le détroit tourne de façon décisive à l'avantage de la RPC et, sans de nouveaux achats d'armes de haut niveau, comme les F-16 C/D souhaités par Taipei, Taiwan aussi bien que les États-Unis courent le risque de céder tant de terrain à Pékin qu'ils se retrouvent incapables à l'avenir de défendre efficacement l'île ${ }^{(2)}$.

\section{L'économie de Taiwan sous la présidence de Ma}

L'économie taiwanaise a été au plus bas depuis l'arrivée au pouvoir du KMT, influençant largement l'opinion sur les capacités et l'efficacité de Ma et de son gouvernement. Ma a été élu sur un programme de forte croissance et de compétence économiques. Toutes deux ont été absentes pendant les deux premières années de son gouvernement, avant même la crise financière internationale. Le bilan du gouvernement de $\mathrm{Ma}$ contraste aujourd'hui de manière négative avec la situation dont il avait hérité du DPP. Contrairement à la propagande électorale du KMT peignant un paysage économique de désastre avant 2008, l'économie taiwanaise sous le gouvernement du DPP était en réalité très performante en termes de croissance économique, d'exportations, de réussites technologiques, de qualité des produits et de création de richesse, finissant avec une forte croissance de
$6,88 \%$ en glissement annuel au premier trimestre 2008 après $5,98 \%$ pour l'ensemble de $2007^{(22)}$. Cela fait cependant plusieurs années que l'économie taiwanaise connait des problèmes d'accroissement des inégalités sociales, de stagnation des revenus salariaux, de baisse du pouvoir d'achat et de délocalisation majeure vers la Chine d'industries taiwanaises cherchant à réduire leurs coûts de production. Tous représentent de profonds problèmes structurels mettant au jour une redistribution des richesses déficiente et l'insuffisance des investissements intérieurs, et requièrent une difficile transformation et amélioration de l'ensemble de la structure et des politiques socio-économiques ${ }^{(23)}$. Alors que l'économie redémarre, le gouvernement du KMT devra s'atteler à résoudre les mêmes problèmes, rendus plus aigus encore par la crise économique, qui ont plombé les performances économiques par ailleurs solides du DPP, ou bien risquer une sanction similaire de l'électorat aux prochaines élections.

L'économie taiwanaise est si liée au marché américain que l'impact de la crise américaine des subprimes de 2007 sur Taiwan s'est fait sentir avant même l'effondrement financier de septembre $2008^{(24)}$. La crise a ensuite frappé Taiwan de plein fouet au cours du quatrième trimestre de 2008 menant à une croissance de $0,73 \%$ en 2008 et à la pire récession de l'histoire moderne de Taiwan en 2009, même si celle-ci a été finalement limitée sur l'année à -1,91 \% grâce à un début de reprise au quatrième trimestre. Le chômage a grimpé de 3,9\% au début de 2008 à plus de $6 \%$ à la mi2009, le plus haut niveau jamais atteint, alors que le PIB par habitant pour 2009 retombait à son niveau de 2006, aux alentours de 17000 dollars américains ${ }^{(25)}$. Le gouvernement de Ma Ying-jeou ne peut être rendu responsable de l'impact de la récession mondiale sur l'économie taiwanaise. Mais

19. Cf. Russell Hsiao "China-Taiwan Up Missile Ante », China Brief, vol. 10, n 7, 1er avri 2010 ; Richard Fisher, « The Implications of China's Naval Modernization for the United States ", International Assessment and Strategy Center, 11 juin 2009, http://www.strategycenter.net/research/publD.199/pub_detail.asp\#.

20. Cf. Alan Romberg, « The Winter of PRC Discontent », art. cit.

21. David A. Shlapak, «Questions of Balance. The Shifting Cross-Strait Balance and Implications for the U.S. », Rand Corporation, CT 343, mars 2010.

22. Cf. Frank Muyard, «Élections 2008 à Taiwan », art. cit., p. 92-95. Concernant les chiffres de 2008-2010, cf. DGBAS, "Key Economic and Social Indicators. July 30, 2010 » (chiffres calculés à partir des prix constants de 2006), http://eng.stat.gov.tw/lp.asp?CtNode $=2191 \&$ CtUnit $=1050 \&$ BaseDSD $=7 \& \mathrm{mp}=5$.

23. Sur l'économie taiwanaise, cf. Philippe Chevalieras, " L'économie taiwanaise après le miracle : restructuration de l'industrie, faiblesses structurelles et défi de la Chine », dans ce numéro.

24. Peter C.Y. Chow, «The Impact of the Financial Tsunami on Taiwan's Economy », in B. Wakefield (éd), Taiwan and the Global Economic Storm, Asia Program Special Report, Woodrow Wilson International Center, $n^{\circ} 143$, octobre 2009, p. 5-12.

25. Cf. chiffres du DGBAS, « Key Economic and Social Indicators. July 30, 2010 », art. cit. 
pour quelqu'un qui avait promis un nouveau miracle économique, son gouvernement était clairement mal préparé et a été peu performant dans sa gestion de la crise, contrairement aux attentes générales et au discours du KMT. Entièrement concentré sur les relations avec la Chine, le gouvernement n'a pas vu la crise arriver, l'a sous-estimée quand elle s'est abattue, et a été inefficace dans ses tentatives de soulager ses effets sur l'économie. Les mesures de relance à court terme qu'il a mises en place ont été pour la plupart coûteuses et inefficientes (comme pour le programme des bons de consommation) ou ont eu des effets négatifs non attendus (notamment le programme de stages pour diplômés qui a fait baisser les salaires d'entrée). Le plan quadriennal massif de dépenses de 26 milliards de dollars américains, prévu dans le programme économique « 12 projets i-Taiwan » de Ma, et comprenant 15 milliards de financement d'infrastructures et 6 milliards pour accroitre la compétitivité industrielle, a été long à se mettre en place et est resté sans grand effet en raison des lenteurs administratives classiques et de la rigidité des pratiques budgétaires. Le manque d'investissements publics en 2008 et 2009 résulte aussi ironiquement de la politique de la terre brûlée menée par le KMT au parlement avant 2008 , où pendant des années il mis à profit sa majorité pour bloquer ou réduire l'ampleur des programmes majeurs d'infrastructures proposés par le DPP et tailler régulièrement dans les budgets publics pour des raisons politiciennes.

Dans l'ensemble, la politique économique du KMT est fondée sur une philosophie d'ouverture à la Chine, de libreéchange, de baisse des impôts et de développement des infrastructures avec un fort accent mis sur les grandes sociétés et les entreprises de haute technologie. Le but est de stimuler la production et les exportations et d'inciter les entreprises étrangères et les entreprises taiwanaises basées à l'étranger à investir dans le pays avec l'idée que cela générera de hauts taux de croissance et d'emploi. D'importantes réductions d'impôts ont été accordées aux entreprises et aux particuliers en 2009 et 2010, mais sans mesure de compensation pour rééquilibrer la diminution des recettes de l'État, engendrant ainsi une aggravation du déficit budgétaire et de la dette publique qui inquiète la plupart des experts économiques ${ }^{(26)}$. La pression fiscale à Taiwan a atteint son plus bas niveau historique et, à $12 \%$ du PIB, se situe bien en dessous de celle d'économies développées similaires. Ces mesures accentuent également les inégalités de revenus et font peser l'essentiel de la pression fiscale sur les classes moyennes salariées, tandis que les taxes foncières sont sousévaluées et que les gains sur les transactions financières et immobilières sont quasiment non imposés ${ }^{(27)}$. Parallèlement, la spéculation immobilière à Taiwan, particulièrement dans la région de Taipei, en partie alimentée par le rapatriement de capitaux offshore et d'importants montants de capitaux improductifs, a créé une bulle et a rendu les prix de l'immobilier inaccessibles pour la classe moyenne, accentuant l'insatisfaction vis-à-vis des politiques du gouvernement perçues comme favorisant les plus aisés ${ }^{(28)}$.

Pendant les deux dernières années, le gouvernement de $\mathrm{Ma}$ semblé tout parier sur " la potion magique chinoise ", et son action a été essentiellement orientée sur les relations entre Taiwan et la Chine plutôt que vers la transformation de l'économie nationale, le soutien à la consommation et au pouvoir d'achat à long terme ou la diversification des exportations. Malheureusement pour Ma, sa politique chinoise n'a pas apporté à la population grand chose des profits économiques promis. Si le plan de relance gouvernemental chinois de 2009 a aidé à amortir le choc de l'effondrement des marchés mondiaux, la valeur des exportations taiwanaises vers la Chine aussi bien que la part taiwanaise des importations chinoises ont chuté en $2009^{(29)}$, et la reprise actuelle des exportations est en majorité, encore une fois, liée au retour de la croissance aux États-Unis et dans d'autres marchés développés ainsi que dans l'ensemble des économies émergentes. Le boom commercial promis à la suite des liaisons directes et de l'arrivée de touristes chinois ne s'est pas matérialisé. L'ouverture de liaisons aériennes régulières (au nombre de 370 par semaine à compter de septembre 2010) et la baisse des coûts de transport des passagers et des marchandises avec la Chine réjouissent les touristes et les hommes et femmes d'affaires taiwanais basés sur le continent (Taishang), mais ne touchent pas la majorité de la population qui voyage peu ou pas du tout en Chine ${ }^{(30)}$. Les bénéfices économiques de l'arrivée de touristes chinois sont globalement restés un mirage pour 2008 et une grande partie de 2009. Bien moins nombreux qu'annoncés, ils se sont révélés peu dépensiers et viennent pour la plupart dans des circuits de

26. "Stunned by the So-Called Tax Reform », Commercial Times, 20 avril 2010; "Auditor Warns of Worsening National Debt Situation », Central News Agency, 28 juillet 2010.

27. Monique Hou, «Tax System Controversy. Taiwan, "Island of Inequity"? », CommonWealth Magazine, $\mathrm{n}^{\circ}$ 445, 22 avril 2010.

28. Hsiang-Yi Chang, "What's Fueling Taiwan's Land Price Explosion », CommonWealth Magazine, $n^{\circ} 445,29$ avril 2010.

29. "Taiwanese Products Lose Market Share in China in 2009: MOEA », Central News Agency, 30 janvier 2010.

30. Dans un sondage de $2009,61,6 \%$ des personnes interrogées déclaraient ne jamais être allées en Chine, 10,1\% y être allées seulement une fois, tandis que $12,7 \%$ s'y étaient rendues plus de cinq fois, cf. " Survey Topic: People's Views on the Chinese Mainland ", Want Daily, 11 août 2009, http://www.kmt.org.tw/english/page.aspx?type=article\& mnum $=114 \&$ anum $=6703$. 
groupes bon marché. Les chiffres pour 2010 semblent finalement décoller et leur impact économique réel sera mieux évalué d'ici quelques années. Cependant, à moins que leur nombre n'augmente de façon exponentielle, les touristes chinois ne constitueront pas une source majeure de revenus pour Taiwan, une réalité connue de tous les économistes depuis le début.

Les autres accords économiques avec la Chine sont également clairement dirigés vers les grandes entreprises et les sociétés financières depuis longtemps désireuses de s'introduire davantage en Chine, que ce soit pour réduire leurs coûts ou pour pénétrer le marché chinois. Comme deux décennies de relations économiques inter-détroit le montrent toutefois, cette expansion bénéficie plus aux hommes d'affaires, aux managers et au personnel hautement qualifié qu'au reste de la population. Le flot continu de délocalisations industrielles vers la Chine a déjà complètement ou partiellement transféré des secteurs entiers de l'économie (chaussures, jouets, textile, cuir, céramique, appareils électriques, petit électronique, ordinateurs), laissant principalement dans l'île la production de produits plus chers et plus sophistiqués, et engendrant une augmentation du chômage et une pression à la baisse sur les salaires à Taiwan ${ }^{(31)}$. Le risque est maintenant d'assister à un accroissement de ces délocalisations en Chine tant dans l'industrie traditionnelle que dans la haute technologie, que ce soit pour s'en servir comme base d'exportations (vers le reste du monde ou vers Taiwan) ou pour étendre leur présence sur le marché chinois. Les statistiques récentes semblent confirmer cette tendance. Depuis la première moitié de 2010, l'économie montre des signes de reprise à la suite du regain des exportations qui s'approchent de leurs plus hauts niveaux d'avant la récession ${ }^{(32)}$. Mais si la proportion des exportations taiwanaises vers la Chine continue d'augmenter, atteignant le seuil historique de $44,2 \%$ des exportations totales en avril 2010, la moitié de ces exportations est constituée de produits semi-finis qui sont traités et assemblés en Chine, la plupart du temps par des sociétés taiwanaises, pour être réexportés vers les marchés des pays développés ${ }^{(33)}$. La structure des commandes d'exportations taiwanaises montre en fait que la part des commandes pour des produits fabriqués par des sociétés taiwanaises en Chine augmente continuellement par rapport à ceux fabriqués à Taiwan, générant des profits accrus pour les entreprises taiwanaises mais peu de bénéfices pour les travailleurs taiwanais ${ }^{(34)}$.

Ces questions ont occupé une place centrale dans le débat autour de l'ECFA, qui s'est rapidement transformé en combat politique entre le KMT et le DPP, l'ECFA alliant les plus chers espoirs et les pires inquiétudes quant à l'intégration économique et le rapprochement politique avec la Chine. Incapable jusqu'à présent de démontrer les bénéfices concrets de liens plus étroits avec la Chine, le gouvernement de Ma concentre tous ses efforts sur l'ECFA qu'il présente comme le seul moyen de faire sortir Taiwan de l" " isolement " économique et de l'oubli. Selon lui, l'ECFA constitue un accord-cadre limité de libéralisation du commerce et des investissements sans signification politique et visant à réduire les tarifs douaniers et à protéger les investissements et les droits de propriété intellectuelle taiwanais en Chine. Ses objectifs sont de faciliter l'accès au marché chinois à une série d'industries et de services taiwanais inclus dans une liste dite de " première récolte " (principalement la pétrochimie, les machines-outils, la sous-traitance automobile, les équipements de transport, et la finance) ${ }^{(35)}$, de protéger l'économie de l'impact de l'accord de libre-échange ASEAN-Chine mis en œuvre en 2010 et du futur accord ASEAN + 3 (Chine, Corée, Japon) attendu pour 2011 ou 2012, et enfin d'encourager la signature d'accords additionnels de libre-échange entre Taiwan et les pays aujourd'hui réticents à indisposer la Chine en s'engageant dans des ententes économiques avec l'île. Le KMT a également promis de soutenir les secteurs de l'économie menacés par l'ouverture de Taiwan aux produits chinois à bas prix, avec la création d'un fonds de modernisation industrielle et de protection sociale de 95 milliards de dollars taiwanais (3 milliards de dollars américains) sur dix ans, et assuré qu'aucun nouveau produit agricole chinois ne serait autorisé à l'importation.

De son côté, le DPP critique l'ECFA sur de nombreux points. Il a d'abord dénoncé le manque d'informations détaillées sur le contenu de l'accord et le manque de transparence sur les négociations. Il s'oppose également à la trop grande dépendance de l'économie taiwanaise envers la RPC induite par l'ECFA, alors que Taiwan est déjà l'économie la plus intégrée à la Chine, et critique les intentions

31. Cf. T. J. Cheng, "China-Taiwan Economic Linkage: Between Insulation and Superconductivity ", in Nancy Tucker (éd.), Dangerous Straits. The U.S.-Taiwan-China Crisis, New York, Columbia University Press, 2005, p. 93-130.

32. CEPD, Taiwan's Economic Situation and Outlook, juin 2010, http://www.cepd.gov.tw/ encontent/.

33. "Record High Percentage of Taiwan's Exports Sent to China in April », Central News Agency, 7 mai 2010; Jonathan Adams, "Weighing the Costs in Asian Trade Talks », New York Times, 12 mai 2010.

34. " March Export Orders Hit All-Time High », United Daily News, 21 avril 2010.

35. Pour le texte intégral de l'accord, voir le site du MAC : www.mac.gov.tw/ct.asp?xltem= $85781 \& \mathrm{ctNode}=6727 \& \mathrm{mp}=1$; pour une analyse de la liste des « primeurs » et sa négociation, voir aussi Alan Romberg, « Ma at Mid-Term: Challenges for Cross-Strait Relations », China Leadership Monitor, n 33, été 2010. 
politiques d'unification de Pékin et du KMT qui se cachent derrière l'ECFA. Le DPP dénonce aussi le fait que l'ECFA ne profitera qu'aux grandes entreprises, menacera les PME et ouvrira la porte à de plus nombreuses importations chinoises et à plus de délocalisations d'usines vers la Chine, aggravant davantage les écarts de richesse et augmentant le chômage et les inégalités sociales à Taiwan. Les critiques de l'accord soulignent que la hausse générale des exportations taiwanaises dans la première moitié de 2010 montre que Taiwan n'a pas besoin de l'ECFA pour accroitre ses exportations vers la Chine et que, contrairement aux prédictions alarmistes du gouvernement, l'économie est dans l'ensemble peu affectée par l'accord de libre-échange ASEAN-Chine, les produits taiwanais n'étant pas en concurrence directe avec les marchandises de l'ASEAN en Chine ni avec les produits chinois dans l'ASEAN. Ils attirent également l'attention sur le fait que d'après les règles de l'OMC, un tel accord-cadre de libre-échange doit conduire dans les dix ans à l'abolition des barrières douanières sur plus de $90 \%$ des produits échangés entre les deux parties ${ }^{(36)}$. Le camp vert a donc appelé à la tenue d'un referendum sur l'EFCA en plus d'un examen complet de l'accord par le Parlement, à l'opposé des approbations législatives automatiques et hâtives des précédents accords avec la Chine.

Le gouvernement a bien du mal à répondre à ces critiques et a dû reconnaitre que l'EFCA ferait des gagnants comme des perdants (essentiellement dans les industries traditionnelles et les PME), mais il s'oppose à un referendum et a promis de laisser au Parlement le pouvoir et le temps d'examiner l'accord final. Après plus d'une année d'intense promotion de l'EFCA par le gouvernement et un débat télévisé sur le sujet entre Ma Ying-jeou et la présidente du DPP Tsai Ingwen en avril 2010, les sondages indiquaient que la plupart des Taiwanais ne connaissaient ni ne comprenaient le contenu de l'accord et qu'aucune majorité claire ne se dessinait pour ou contre l'EFCA ${ }^{(37)}$. Même après la signature de l'accord, un sondage indépendant a montré que seuls $47 \%$ des Taiwanais le soutiennent ${ }^{(38)}$. Une majorité de personnes était aussi en faveur de la tenue d'un referendum sur l'ECFA, y compris au sein du camp bleu ${ }^{(39)}$. Après le rejet en juin et en août de ses projets de referendum par la Commission gouvernementale d'examen référendaire au motif d'obscures questions de procédure, le camp vert prévoit de soumettre une nouvelle proposition à l'automne $2010^{(40)}$.

LECFA a finalement été adopté par le Parlement le 17 août 2010 selon la procédure préférée du gouvernement d'une approbation globale de l'accord plutôt que par un vote sur chaque article comme le demandait le DPP ${ }^{(4)}$. L'accord doit entrer en vigueur en septembre 2010, la réduction des tarifs douaniers commençant en janvier 2011. Aucun impact économique réel de l'ECFA n'est attendu pour 2010, et les bénéfices globaux de l'accord sont encore difficiles à évaluer. Toutefois, les recherches sur d'autres accords de libreéchange ou de réductions de tarifs douaniers ont montré qu'ils avaient tous tendance à profiter aux industries à bas coûts des pays en développement et aux élites économiques de toutes les parties concernées, tandis que les classes moyennes et populaires des pays développés souffraient le plus et que les écarts de revenus augmentaient ${ }^{(42)}$. Si la signature de l'ECFA peut réduire l'opposition politique de Pékin envers les accords commerciaux internationaux de Taiwan et conduire à la signature d'accords de libre-échange entre Taiwan et ses autres partenaires économiques régionaux, l'Union européenne, et, surtout, les États-Unis, comme promis par le gouvernement de Ma, l'EFCA pourrait malgré tout être vu comme un outil utile à la promotion des intérêts politiques et économiques internationaux taiwanais.

Du point de vue politique cependant, un rapport interne du Centre du budget et du Bureau de recherche sur les affaires juridiques du Yuan législatif taiwanais publié fin juillet, tend à légitimer les principales inquiétudes de l'opposition en mettant l'accent sur les risques économiques et sécuritaires de l'ECFA qui pourraient conduire à un "siège économique " et menacer la souveraineté de Taiwan ${ }^{(43)}$. Confir-

36. Pour une analyse critique des aspects économiques et politiques de l'ECFA, voir Chen Po-chih (éd.), ECFA: bu neng shuo de mimi?, Taiwan zhiku, 2010 (ECFA: Unspeakable Secrets?), Taiwan ThinkTank, 2010.

37. Cf. Sondage TVBS sur le referendum sur l'ECFA, 31 mai 2010. Pour ce sondage et d'autres sondages TVBS cités dans cet article, cf. www.tvbs.com.tw/news/poll_center/ index.html.

38. Global Views Survey Research Center, « Survey on Signed ECFA etc. », 28 juillet 2010. Sauf si cela est précisé, pour ce sondage et les autres sondages de Global Views cités dans cet article, cf. http://www.gvm.com.tw/gvsrc/eng/index.asp.

39. $55 \%$ de la population totale et $47 \%$ au sein des sympathisants du camp bleu. Cf. Sondage TVBS sur le referendum sur l'ECFA, op. cit.

40. "TSU Vows to Go On After Poll is Rejected », Taipei Times, 12 août 2010.

41. " ECFA Receives Legislative Approval », Taipei Times, 18 août 2010. Voir aussi Jerome A. Cohen et Yu-Jie Chen, « Taiwan's Leaders Should Welcome Review of Cross-strait Pacts ", South China Morning Post, 7 juillet 2010.

42. «Bracing for Uneven Distribution of Wealth », Commercial Times, 3 mai 2010 ; R. Scott, C. Salas et B. Campbell, Revisiting NAFTA. Still Not Working for North America's Workers, Economic Policy institute, Briefing Paper 173, 28 septembre 2006. Pour l'impact de l'accord américain pour des relations commerciales normales permanentes avec la Chine sur l'économie américaine, cf. Harold Meyerson, " A Marriage Made in China », Washington Post, 18 novembre 2009.

43. Cf. Martin Williams, " Taiwan Legislative Report Warns of Danger in China Trade Deal », Kyodo, 28 juillet 2010. Flora Wang, « Report from Legislative Agency Details the Crossstrait Political Risk of ECFA », Taipei Times, 29 juillet 2010. Le rapport se base en partie sur une étude de terrain menée en mai 2010 et sur l'analyse de l'accord de libreéchange entre Hong Kong et la Chine, le CEPA, et ses conséquences sur l'économie, la politique et la liberté de la presse à Hong Kong. L'opposition a accusé le KMT de bloquer la publication du rapport d'évaluation en juin afin de ne pas gêner la signature de l'ECFA. 
mant la nature politique de l'EFCA, au moins pour Pékin, le rapport souligne que l'accord est un élément clé de la stratégie chinoise d'unification avec Taiwan et que Pékin entend l'utiliser comme un levier pour augmenter la dépendance économique de Taiwan envers la Chine et faire pression en faveur de pourparlers sur l'unification.

\section{Performance gouvernementale et jugement de l'opinion}

À mi-mandat, l'incapacité de Ma Ying-jeou à honorer ses promesses d'une rapide amélioration économique et la prise de conscience collective, y compris par $\mathrm{Ma}$, que son programme 6-3-3 n'est qu'un lointain rêve, ont des répercussions majeures sur ses taux d'approbation et de confiance au sein de l'opinion publique. Sa politique de rapprochement avec la Chine a aussi suscité de fortes oppositions dans le camp vert et l'électorat du centre. Mais d'autres faux-pas importants de la part du président et de son gouvernement ont contribué à ternir davantage leur image et expliquent leur faible popularité. Le mécontentement est largement répandu et ne se limite pas à l'opposition, les critiques directes et les éditoriaux acerbes fusant des rangs du KMT comme des médias favorables au camp bleu.

Le premier objet de la critique publique est la série de crises et leur mauvaise gestion par le gouvernement depuis 2008 qui ont révélé un mélange d'incompétence, d'arrogance et de négligence de la part des autorités de l'État. La réponse désastreuse de Ma et de son gouvernement au typhon Morakot d'août 2009 et aux inondations qui l'ont suivi, les pires qu'ait connu Taiwan en 60 ans faisant plus de 600 victimes, a été l'exemple le plus frappant de l'incapacité du gouvernement à réagir efficacement et rapidement à un défi majeur. La crise fut aussi aggravée par le désintérêt apparent du gouvernement pour les problèmes et l'opinion de la population, sa réticence à accepter l'aide internationale, et une tendance à fuir ses responsabilités en blâmant les autres pour ses propres insuffisances. Mais les réactions déficientes à des typhons moins meurtriers et au scandale du lait contaminé à la mélamine importé de Chine pendant l'été 2008, ainsi que le fiasco intégral de la ré-autorisation des importations américaines de bœuf en violation d'une résolution législative et sans tenir compte des inquiétudes de l'opinion à l'automne 2009 , sont autant d'autres cas de mauvaise gestion qui ont sérieusement entamé la confiance publique envers le gouvernement. Les conséquences du typhon Morakot ont provoqué la démission du technocratique Premier ministre Liu Chaohsuan et un remaniement important du gouvernement au- jourd'hui conduit par Wu Den-yi, un politicien du KMT plus près des gens. Depuis, une série de couacs administratifs, de mesures contestées, de revirements et de volte-face politiques ainsi qu'une déception générale envers les performances gouvernementales ont toutefois continué à saper la crédibilité et le taux de confiance du président et de son équipe au sein de l'opinion.

L'échec de Ma à honorer ses promesses de réconciliation nationale à l'intérieur du pays est une autre raison de la déception publique à son égard. Au lieu de tendre la main à l'opposition et de tenter d'esquisser un consensus en matière de politiques économiques et sociales ou bien sur la Chine et la politique étrangère, Ma et son gouvernement sont restés globalement sourds aux critiques et aux propositions, alimentant ainsi la polarisation politique de la société et rejetant les membres du camp vert qui, après leur défaite cuisante, auraient probablement été prêts à participer à un grand débat sur la politique nationale ${ }^{(44)}$. Pire, le gouvernement de $\mathrm{Ma}$ s'est engagé dans ce qui ne peut être qu'appelé une politique revancharde et de chasse aux sorcières contre de nombreux anciens responsables et fonctionnaires des gouvernements DPP par le biais d'enquêtes et de poursuites judiciaires sur de présumés scandales de corruption, conduisant observateurs et experts judiciaires étrangers à s'inquiéter sérieusement du comportement des fonctionnaires de justice et de l'usage politique de la justice sous le régime du $\mathrm{KMT}^{(45)}$. La saga du procès de l'ancien président Chen Shui-bian s'est conclue sur un verdict de réclusion criminelle à perpétuité pour corruption (peine réduite à 20 ans en appel en juin 2010) sans preuve de liens directs avec les malversations financières de son entourage et à un moment où le mauvais usage ou la dissimulation présumés de dons politiques faisaient partie des règles du jeu dans le flou juridique entourant le financement politique taiwanais ${ }^{(46)}$. Le procès a également été entaché par une série d'actions et de décisions douteuses des procureurs et des institutions juridiques concernant les droits de la défense et le droit à un procès équitable ${ }^{(47)}$. Si l'opinion publique croit en un certain degré de culpabilité de Chen Shui-bian, la façon dont son procès

44. Julian Baum, « Who's Listening to Taiwan's People ? », Far Eastern Economic Review, 6 novembre 2009.

45. "Open Letter on Erosion of Justice in Taiwan », Taipei Times, 6 novembre 2008 ; Jerome A. Cohen, «Silence of the Lambs. Taiwan's Top Minds Need to Speak Out on Law Reforms to Help Achieve the Best Democratic Solutions ", South China Morning Post, 11 juin 2009.

46. Jonathan Manthorpe, «Evidence Fails to Support Life Sentence for Taiwan's Former President », Vancouver Sun, 16 septembre 2009

47. William Lowther, « US Academics Decry Unfair Treatment in Chen Trial », Taipei Times, 28 septembre 2009. 
et d'autres poursuites judiciaires de personnalités politiques et d'anciens fonctionnaires proches du DPP ont été conduits par l'administration $\mathrm{Ma}$ a profondément desservi la société et la démocratie taiwanaises et montre la nécessité d'une profonde réforme du système judiciaire pour qu'il puisse atteindre le niveau observé dans des démocraties plus mûres ${ }^{(48)}$. Parallèlement, des projets de loi du ministère de la Justice menaçant certains droits fondamentaux de la défense et des avocats ont conduit les communautés juridique et académique et les groupes civiques à tirer la sonnette d'alarme ${ }^{(49)}$.

D'autres sources majeures d'inquiétude envers le gouvernement touchent aux libertés publiques, à la liberté de la presse et aux dérives de la police. Les actions répressives de l'administration policière avant, pendant et après des manifestations publiques ou des rassemblements privés critiquant le président ou la politique gouvernementale depuis 2008 (et plus particulièrement pendant les manifestations contre la deuxième rencontre Chiang-Chen à Taipei en novembre 2008), la fausse réforme de la loi sur les rassemblements et les manifestations toujours utilisée par les procureurs contre les manifestants politiques et les militants des droits civiques, et d'autres lois portant atteinte aux libertés, ont provoqué la colère non seulement de l'opposition politique mais aussi de la plupart des universitaires et des activistes démocrates ${ }^{(50)}$. De mauvaises habitudes de contrôle et d'intimidation rappelant les années du régime autoritaire $\mathrm{du}$ KMT semblent ainsi de retour et conduisent les Taiwanais à se demander si la pleine démocratie et la liberté dont ils jouissaient pendant les années Chen est en danger ${ }^{(5)}$. Du côté des médias, les tentatives répétées de la part du gouvernement de Ma de contrôler politiquement et d'aligner tous les médias publics (chaines de télévision, Agence centrale de presse) derrière la politique gouvernementale plutôt que d'encourager l'analyse critique et le reportage équilibré qui est le devoir du journalisme libre, ont été condamnées par les défenseurs internationaux de la liberté de la presse ${ }^{(52)}$. L'achat du groupe de presse China Times autrefois contrôlé par le KMT (y compris ses chaînes de télévision) par l'homme d'affaires taiwanais prochinois et basé en Chine Tsai Eng-Meng a aussi soulevé la crainte de voir l'industrie des médias, déjà largement en faveur du KMT, tomber sous l'influence de groupes liés à Pékin, et la liberté de la presse se détériorer au point où une autocensure à la hongkongaise ou à la singapourienne et la propagande autocongratulatrice de puissants médias pro-gouvernementaux saperaient l'échange public d'opinions politiques et sociales ${ }^{(33)}$. L'ONG américaine Freedom House a ainsi si- gnalé pour la deuxième année consécutive le déclin de la liberté de la presse dans l'île qui a chuté de 14 places pour atteindre le $47^{\circ}$ rang mondial et est passée du premier au $8^{\mathrm{e}}$ rang en Asie depuis $2008^{(54)}$.

Le mécontentement public se reflète dans de multiples sondages d'opinion conduits par différents bureaux d'enquêtes, y compris par des médias pro-KMT comme United Daily News, China Times, TVBS et Global Views Magazine, et qui montrent le déclin quasi continu de la popularité de $\mathrm{Ma}$ et de son gouvernement. Selon les sondages de Global Views, le taux d'approbation de Ma Ying-jeou a ainsi chuté dès son investiture, avec un taux d'opinions défavorables s'élevant à 46,2 \% en juin 2008 contre $11,9 \%$ le mois précédent et dépassant déjà son taux d'opinions favorables de $37,8 \%$. Son taux d'approbation est ensuite constamment resté inférieur à $40 \%$. Depuis le typhon Morakot d'août 2009 , il oscille entre 20 et $30 \%$ (28,4\% en juin 2010), contre un taux de désapprobation d'environ $60 \%$. Le haut niveau de confiance avec lequel Ma est arrivé au pouvoir à $55,4 \%$ a également diminué pour atteindre $38,3 \%$ en juin 2010, son plus bas niveau en deux ans, contre $43 \%$ de la population qui ne lui fait pas confiance ${ }^{(5)}$. Ceci démontre que l'insatisfaction envers $\mathrm{Ma}$ est largement partagée, au sein du camp bleu et des électeurs du centre aussi bien que dans le camp vert. La crise de confiance à laquelle son gouvernement est confronté n'est donc pas la simple conséquence des problèmes économiques, mais est aussi alimentée par l'opposition que suscite sa vigoureuse politique prochinoise et la déception envers les performances gouvernementales.

48. Jerome A. Cohen, «A Work in Progress. The Trial of Chen Shui-bian Highlights the Shortcomings of a Legal System in Transition ", South China Morning Post, 17 septembre 2009.

49. Jerome A. Cohen et Yu-Jie Chen, « Under Threat. Taiwan's Criminal Defense Lawyers and China's ", South China Morning Post, 20 janvier 2010; Celia Llopis-Jepsen, « The Backsliding of Judicial Reform », Taipei Times, 19 janvier 2010.

50. Cf. «Wild Strawberries Slam Indictment of NTU Professor », Taipei Times, 12 juin 2009 Loa lok-sin, « Activists, Academics "Surrender" to Protest Law », Taipei Times, 14 juillet 2009 ; Chiu Hei-Yuan, «The Act that Silences Academics », Taipei Times, 13 juillet 2009.

51. Dafydd Fell, «Taiwan's Democracy: Towards a Liberal Democracy or Authoritarianism? » Journal of Current Chinese Affairs, vol. 39, $\mathrm{n}^{\circ}$ 2, 2010, p. 187-201.

52. Reporters sans frontières, «Taiwan. Public Media Independence Threatened, Despite Government Denials ", 17 décembre 2008; William Lowther, "Group Monitoring Taiwan Press », Taipei Times, 6 mai 2009.

53. Shelley Shan, "Analysts Wary of China Times Group's Agenda », Taipei Times, 9 mai 2009 ; Ting-I Tsai, « Move to Replace Taiwan Editor Spurs Talk », Wall Street Journal, 11 janvier 2010.

54. Vincent Y. Chao, « Taiwan's Press Freedoms in Decline, Watchdog Says », Taipei Times, 1er mai 2010.

55. Cf. Global Views Survey Research Center, « Survey on President Ma Ying-jeou's Approval Rating », 21 juin 2010. Les sondages TVBS font état des mêmes tendances avec un sondage du 19 mai 2010 rapportant un taux d'opinions défavorables de $47 \%$ contre seulement $33 \%$ d'opinions favorables. 
Tableau 1. Résultats généraux des élections régionales taiwanaises 2001-2009 (avec comparaisons pour les 17 circonscriptions de 2009)

*Elue comme candidate indépendante, la chef du comté de Taitung a rejoint le KMT après sa victoire.

Tableau : Frank Muyard. Source : Commission électorale centrale

Élections régionales et compétition politique à Taiwan

Cette insatisfaction générale s'est logiquement répercutée sur les nombreuses élections locales tenues depuis 2008. On s'intéressera d'abord aux élections régionales de décembre 2009 puis aux élections législatives partielles de ces deux dernières années afin d'évaluer les changements de fortune du KMT et du DPP.

\section{Les élections régionales de décembre 2009}

Les $16^{\text {e }}$ élections régionales pour les postes de chef de comté et de maire de ville moyenne ont eu lieu le 5 décembre 2009 en conjonction avec les élections pour les conseils municipaux et de comté ainsi que pour les maires de bourgs selon la formule trois-en-une inaugurée en 2005. Cependant, seuls 17 des 23 villes et comtés en jeu en 2005 étaient cette fois concernés du fait de la décision gouverne- mentale de juillet 2009 de fusionner et promouvoir certains comtés et villes au rang administratif de municipalité spéciale réservé auparavant aux seules villes de Taipei et de Kaohsiung. Taiwan compte par conséquent maintenant cinq municipalités spéciales pour lesquelles les élections pour les postes de maire et de conseillers municipaux auront lieu en novembre 2010 : Taipei, la nouvelle ville de Sinbei (anciennement le comté de Taipei), et les nouvelles villes de Taichung, Tainan et Kaohsiung créées en fusionnant les anciennes villes et comtés du même nom. L'électorat des 17 villes et comtés restants représente seulement $40 \%$ de la population taiwanaise contre $60 \%$ pour les cinq municipalités spéciales. Les élections de décembre 2009 ont néanmoins été politiquement significatives à deux égards. Tout d'abord elles ont donné des indications sur le poids respectif des deux principaux partis et sur leur capacité à mobiliser leurs sympathisants et leurs réseaux locaux, particulièrement pour le KMT dont la machine électorale est particulièrement puissante lors des élections locales. Deuxièmement, même si 
elles étaient locales par nature, elles ont constitué le premier test électoral à grande échelle et d'ampleur nationale pour le nouveau gouvernement du KMT ainsi que pour un DPP en cours de restructuration sous la direction de Tsai Ing-wen ${ }^{(56)}$. Le résultat des élections a été une victoire symbolique pour le DPP et un revers relatif pour le KMT (voir tableau 1). Sur les 14 villes et comtés contrôlés précédemment par le KMT, une est revenue au DPP et une autre à un candidat indépendant dissident du KMT. Si le nombre de sièges remportés est toujours largement favorable au KMT, les résultats en termes de votes et en pourcentages montrent une nette progression du DPP. Comparés aux résultats de 2005 (dans les mêmes 17 districts), le camp vert conduit par le DPP a enregistré une progression totale de ses suffrages de 5,82\% pour atteindre $45,32 \%$, tandis que le camp bleu mené par le KMT a perdu $4,3 \%$ pour tomber à $47,88 \%$, et que le score des indépendants a diminué de $1,52 \%$ pour atteindre $6,8 \%$. Ceci constitue les meilleurs résultats jamais atteints par le DPP lors d'élections régionales dans ces districts, tandis que le KMT enregistre son pire résultat depuis 1997 (année où un grand nombre de candidats dissidents du KMT ont faussé le vote et mené à la perte de nombreux sièges en faveur du DPP). L'écart entre les deux camps s'est réduit à seulement $2,5 \%$, un net succès pour le camp vert, même si la plupart des voix indépendantes peuvent être considérées comme pro-camp bleu. Comparée aux élections présidentielles de 2008 dans les mêmes districts, le pourcentage des voix du DPP augmente également du même chiffre de $5,8 \%$, tandis que le KMT enregistre une baisse de $12,63 \%$ par rapport aux $61 \%$ remportés par Ma Ying-jeou. L'analyse du nombre de voix montre les mêmes tendances. Avec une plus faible participation et 93000 suffrages valides en moins par rapport à 2005, le DPP a réussi à remporter 218000 votes supplémentaires, le KMT en a perdu plus de 236000 retombant au niveau de l'ensemble du camp bleu en 2001, et les indépendants ont reculé de 74000 voix. De plus, et de façon peut-être plus importante pour les élections futures, le DPP a systématiquement augmenté ses suffrages dans des régions traditionnellement pro-bleu (voir tableau 2). Des comtés considérés acquis au KMT comme Taoyuan, Taitung ou Penghu, ont été plus disputés que prévu, avec d'importants progrès également enregistrés à Keelung et dans la ville de Hsinchu. Dans le même temps, les sortants du DPP à Yunlin et Pingtung ont été réélus avec des majorités écrasantes de plus de $60 \%$, et une progression à deux chiffres par rapport à 2005. Une abstention plus forte alliée aux progrès du DPP montre que le KMT n'a pas réussi à mobiliser sa base tandis qu'une proportion significa- tive d'électeurs du milieu s'est tournée vers le DPP. Le retour du comté d'Ilan, longtemps gouverné par le Tangwai et le DPP, dans le giron du camp vert après quatre ans aux mains du KMT a aussi été une victoire symboliquement importante. De même, la perte du bastion bleu du comté de Hualien au profit d'un ancien parlementaire du PFP qui s'est présenté comme indépendant après s'être vu refusé l'investiture du KMT pour ses démêlés judiciaires et auquel Ma Ying-jeou s'était publiquement opposé, constitue une claire défaite personnelle pour le président ${ }^{(57)}$.

Au total, le DPP a également obtenu un meilleur résultat lors de ces élections que lors des élections de 2005 qui impliquaient 23 villes et comtés (+ 2,06\%). De plus, le fait qu'en 2005 les résultats globaux du DPP dans les 23 districts étaient de 3,76\% supérieurs à ses résultats dans les 17 circonscriptions (en jeu en 2009) indique que sa base électorale est plus importante dans les 6 villes et comtés qui ont fusionné ou été promus au rang de municipalités spéciales. Une des causes de ceci est la teinte plus rurale de ces 17 circonscriptions alors que les agglomérations de Taipei, Taichung, Tainan et Kaohsiung, où le DPP enregistre généralement de meilleurs scores, sont plus urbanisées, ainsi que le poids de la région méridionale de Tainan et Kaohsiung où le soutien pour le camp vert est traditionnellement plus fort. Le DPP aurait donc peut-être pu remporter les élections régionales en nombre de suffrages si elles s'étaient tenues dans l'ensemble des anciennes 23 circonscriptions.

Plus généralement, et même s'il faut éviter de trop extrapoler sur le sens national d'élections locales, le scrutin de décembre constitue un avertissement fort de la part de l'électorat à l'administration du KMT concernant la façon dont elle gouverne le pays. Le KMT l'a reconnu tout en rejetant tout changement d'orientation politique autre que d'améliorer la communication de son travail et de mieux tenir compte des besoins de la population. Comme les élections partielles suivantes l'ont prouvé, cette réponse d'ordre cosmétique n'a apparemment été suffisante pour changer l'opinion des électeurs. De son côté le DPP a accueilli les résultats avec pru-

56. Les résultats globaux des élections régionales de 2009 ne peuvent être directement comparés aux élections précédentes puisque différents électorats sont en jeu. Les tableaux 1 et 2 présentent donc les résultats passés reconstitués pour les seuls 17 villes et comtés concernés en 2009.

57. Pour les deux autres scrutins locaux du 5 décembre, les résultats montrent une domination classique du KMT et des indépendants, même si le DPP enregistre une augmentation du nombre de ses élus. Lors des élections pour les conseils municipaux et de comté, le KMT a remporté 43,94 \% des suffrages, le DPP $24,41 \%$ et les indépendants et les autres partis $31,65 \%$. Lors des élections pour les maires de petites villes et de bourgs, le KMT a remporté 121 postes (avec 48,82 \% des voix), le DPP 34 postes (avec $20,04 \%$ ) et les indépendants 56 postes (avec 31,14\%). Source: Commission Electorale Centrale. 
Tableau 2. Résultats des élections régionales taiwanaises de 2005 et de 2009 par circonscriptions (uniquement les 17 villes et comtés de 2009)

\begin{tabular}{|c|c|c|c|c|c|c|c|c|}
\hline \multirow[b]{2}{*}{ Districts } & \multicolumn{4}{|c|}{ Elections régionales de 2005} & \multicolumn{4}{|c|}{ Elections régionales de 2009} \\
\hline & $\begin{array}{c}\text { Electeurs } \\
\text { inscrits I } \\
\text { participation \% }\end{array}$ & $\begin{array}{c}\text { KMT } \\
\text { (Camp bleu) } \\
\text { voix / \% }\end{array}$ & $\begin{array}{c}\text { DPP } \\
\text { (Camp vert) } \\
\text { voix } / \%\end{array}$ & $\begin{array}{l}\text { Indépendants } \\
\text { voix / \% }\end{array}$ & $\begin{array}{c}\text { Electeurs } \\
\text { inscrits I } \\
\text { Participation \% }\end{array}$ & $\begin{array}{c}\text { KMT (Bleu) } \\
\text { voix } / \% \text { I } \\
2009 / 2005 \% \\
\text { variation }\end{array}$ & $\begin{array}{c}\text { DPP }(\text { Vert) } \\
\text { voix } / \% / \\
2009 / 2005 \% \\
\text { variation }\end{array}$ & $\begin{array}{l}\text { Indépendants } \\
\text { voix / \% }\end{array}$ \\
\hline $\begin{array}{r}\text { Total } 17 \\
\text { circonscriptions } 2009\end{array}$ & & $\begin{array}{c}2331337 \\
52,18\end{array}$ & $\begin{array}{c}1764734 \\
39,5\end{array}$ & $\begin{array}{c}371868 \\
8,32\end{array}$ & $\begin{array}{c}7051039 \\
63,34\end{array}$ & $\begin{array}{c}2094518 \\
47,88 \\
-4,3\end{array}$ & $\begin{array}{c}1982518 \\
45,32 \\
5,82\end{array}$ & $\begin{array}{c}297500 \\
6,8\end{array}$ \\
\hline Ilan & $\begin{array}{c}340550 \\
70,25\end{array}$ & $\begin{array}{c}121463 \\
51,39\end{array}$ & $\begin{array}{c}112853 \\
47,75\end{array}$ & $\begin{array}{c}2020 \\
0,85\end{array}$ & $\begin{array}{c}351858 \\
70,68\end{array}$ & $\begin{array}{c}112469 \\
45,74 \\
-5,65\end{array}$ & $\begin{array}{c}133394 \\
54,26 \\
6,51\end{array}$ & \\
\hline Keelung & $\begin{array}{c}292330 \\
64,05\end{array}$ & $\begin{array}{c}76162(124094) \\
41,14(67,04)\end{array}$ & $\begin{array}{c}2771(61014) \\
1,5(32,96)\end{array}$ & & $\begin{array}{c}299960 \\
53,04\end{array}$ & $\begin{array}{c}86001 \\
55,11 \\
-11,93 \\
\end{array}$ & $\begin{array}{c}65673 \\
42,08 \\
9,12 \\
\end{array}$ & $\begin{array}{r}4391 \\
2,81\end{array}$ \\
\hline Taoyuan & $\begin{array}{c}1319334 \\
61,77\end{array}$ & $\begin{array}{c}488979 \\
60,84\end{array}$ & $\begin{array}{c}307965 \\
38,32\end{array}$ & $\begin{array}{c}6784 \\
0,84\end{array}$ & $\begin{array}{c}1437190 \\
53,73\end{array}$ & $\begin{array}{c}396237 \\
52,22 \\
-8,62\end{array}$ & $\begin{array}{c}346678 \\
45,69 \\
7,37\end{array}$ & $\begin{array}{c}15807 \\
2,08\end{array}$ \\
\hline Ville de Hsinchu & $\begin{array}{c}277182 \\
59,6\end{array}$ & $\begin{array}{c}112221 \\
69,27\end{array}$ & $\begin{array}{c}49777 \\
30,73\end{array}$ & & $\begin{array}{c}298439 \\
56,83\end{array}$ & \begin{tabular}{r|}
92667 \\
55,63 \\
$-13,64$ \\
\end{tabular} & $\begin{array}{l}68822 \\
41,32 \\
10,59 \\
\end{array}$ & $\begin{array}{c}5074 \\
3,05\end{array}$ \\
\hline Comté de Hsinchu & $\begin{array}{c}335906 \\
71,12\end{array}$ & $\begin{array}{c}157012 \\
67,09\end{array}$ & $\begin{array}{c}77037 \\
32,91\end{array}$ & & $\begin{array}{c}369480 \\
69,49\end{array}$ & $\begin{array}{l}97151 \\
38,49 \\
-28,6 \\
\end{array}$ & $\begin{array}{c}77126 \\
30,55 \\
2,36\end{array}$ & $\begin{array}{r}78147 \\
30,96\end{array}$ \\
\hline Miaoli & $\begin{array}{c}411296 \\
69,45\end{array}$ & $\begin{array}{c}134277 \\
47,91\end{array}$ & $\begin{array}{c}83694 \\
29,86\end{array}$ & $\begin{array}{c}62313 \\
22,23\end{array}$ & $\begin{array}{c}425837 \\
68,28\end{array}$ & $\begin{array}{c}181256 \\
63,79 \\
15,88 \\
\end{array}$ & $\begin{array}{c}95469 \\
33,6 \\
3,74 \\
\end{array}$ & $\begin{array}{c}7413 \\
2,61\end{array}$ \\
\hline Changhua & $\begin{array}{c}961048 \\
71,16\end{array}$ & $\begin{array}{c}370790 \\
55,46\end{array}$ & $\begin{array}{c}270949 \\
40,52\end{array}$ & $\begin{array}{c}26887 \\
4,02\end{array}$ & $\begin{array}{c}991741 \\
65,56\end{array}$ & $\begin{array}{c}348341 \\
54,89 \\
-0,57 \\
\end{array}$ & $\begin{array}{c}276897 \\
43,63 \\
3,11 \\
\end{array}$ & $\begin{array}{l}9402 \\
1,48\end{array}$ \\
\hline Nantou & $\begin{array}{c}398903 \\
70,48\end{array}$ & $\begin{array}{c}124777 \\
45,32\end{array}$ & $\begin{array}{c}83503 \\
30,33\end{array}$ & $\begin{array}{l}67064 \\
24,36\end{array}$ & $\begin{array}{c}408006 \\
67,75\end{array}$ & $\begin{array}{c}136951 \\
50,87 \\
5,55 \\
\end{array}$ & $\begin{array}{c}107023 \\
39,75 \\
15,39 \\
\end{array}$ & $\begin{array}{c}25243 \\
9,37\end{array}$ \\
\hline Yunlin & $\begin{array}{c}553486 \\
69,73\end{array}$ & $\begin{array}{c}167690 \\
44,48\end{array}$ & $\begin{array}{c}201192 \\
53,37\end{array}$ & $\begin{array}{c}8125 \\
2,16\end{array}$ & $\begin{array}{c}560207 \\
64,61\end{array}$ & $\begin{array}{c}121832 \\
34,63 \\
-9,85 \\
\end{array}$ & $\begin{array}{c}229958 \\
65,37 \\
12 \\
\end{array}$ & \\
\hline Ville de Chiayi & $\begin{array}{c}196054 \\
70,77\end{array}$ & $\begin{array}{c}74786 \\
54,63\end{array}$ & $\begin{array}{l}62122 \\
45,37\end{array}$ & & $\begin{array}{c}202581 \\
66,96\end{array}$ & $\begin{array}{c}69962 \\
52,2 \\
-2,43 \\
\end{array}$ & $\begin{array}{c}61268 \\
45,71 \\
0,34 \\
\end{array}$ & $\begin{array}{r}2801 \\
2,09\end{array}$ \\
\hline Comté de Chiayi & $\begin{array}{c}422387 \\
71,64\end{array}$ & $\begin{array}{c}109192 \\
37,31\end{array}$ & $\begin{array}{c}183476 \\
62,69\end{array}$ & & $\begin{array}{c}428673 \\
75,29\end{array}$ & $\begin{array}{c}128973 \\
40,67 \\
3,36 \\
\end{array}$ & $\begin{array}{c}177333 \\
55,92 \\
-6,77 \\
\end{array}$ & $\begin{array}{c}10829 \\
3,42\end{array}$ \\
\hline Pingtung & $\begin{array}{c}672781 \\
70,68\end{array}$ & $\begin{array}{c}195928 \\
41,86\end{array}$ & $\begin{array}{c}216200 \\
46,19\end{array}$ & $\begin{array}{c}55980 \\
11,96\end{array}$ & $\begin{array}{c}682475 \\
68,09\end{array}$ & $\begin{array}{c}185384 \\
40,67 \\
-1,19 \\
\end{array}$ & $\begin{array}{c}270402 \\
59,33 \\
13,14 \\
\end{array}$ & \\
\hline Hualien & $\begin{array}{c}258736 \\
61,6\end{array}$ & $\begin{array}{c}66575(104954) \\
42,66(67,25)\end{array}$ & $\begin{array}{c}30988 \\
19,86\end{array}$ & $\begin{array}{c}20113 \\
12,89\end{array}$ & $\begin{array}{c}261214 \\
59,47\end{array}$ & $\begin{array}{l}38603 \\
25,44 \\
-41,81\end{array}$ & & $\begin{array}{c}113127(85532 \\
74,56(56,37) \\
43,48\end{array}$ \\
\hline Taitung & $\begin{array}{c}178785 \\
60,51\end{array}$ & & & $\begin{array}{c}105080(62189) \\
100(59,18)\end{array}$ & $\begin{array}{c}178139 \\
61,88\end{array}$ & $\begin{array}{l}56354 \\
52,59\end{array}$ & $\begin{array}{c}50802 \\
47,41\end{array}$ & \\
\hline Penghu & $\begin{array}{c}70427 \\
72,16\end{array}$ & $\begin{array}{c}25223 \\
50,69\end{array}$ & $\begin{array}{c}23964 \\
48,16\end{array}$ & $\begin{array}{l}572 \\
1,15\end{array}$ & $\begin{array}{c}75033 \\
63,33\end{array}$ & $\begin{array}{c}22664 \\
49,37 \\
-1,32\end{array}$ & $\begin{array}{l}22069 \\
48,07 \\
-0,09\end{array}$ & $\begin{array}{l}1177 \\
2,56\end{array}$ \\
\hline Kinmen & $\begin{array}{l}52039 \\
62,78\end{array}$ & $\begin{array}{l}(17359) \\
(54,28)\end{array}$ & & $\begin{array}{l}14622 \\
45,72\end{array}$ & $\begin{array}{c}72509 \\
53,8\end{array}$ & $\begin{array}{c}14269 \\
37,28\end{array}$ & & $\begin{array}{l}24011 \\
62,72\end{array}$ \\
\hline Lienchiang & $\begin{array}{l}8170 \\
60,99\end{array}$ & $\begin{array}{l}(2592) \\
(52,9)\end{array}$ & & $\begin{array}{c}2308 \\
47,1\end{array}$ & $\begin{array}{l}7697 \\
72,25\end{array}$ & $\begin{array}{l}3135(5404) \\
57,19(98,58)\end{array}$ & & $\begin{array}{c}78 \\
1,42\end{array}$ \\
\hline
\end{tabular}

Vainqueurs en caractères gras et en rouge, rose ou noir selon l'affiliation politique. Variation de pourcentage 2005/2009 pour les camps bleu et vert seulement. Tableau : Frank Muyard. Source : Commission électorale centrale 
dence, les considérant comme une réussite encourageante mais limitée et un regain bienvenu de confiance pour le parti après le marasme de la défaite de 2008. Enfin, il faut noter que les élections ont été entachées par des achats de voix à grande échelle, principalement par des candidats du KMT, conduisant les médias à parler d'élections les plus sales de l'histoire, et faisant apparaitre les slogans anti-corruption de Ma Ying-jeou comme creux si ce n'est hypocrites $^{(58)}$.

\section{Les élections législatives partielles de 2009 et 2010}

Dix élections législatives partielles se sont tenues depuis 2008, dont cinq après des décisions de justice invalidant le premier scrutin pour achat de voix ou inéligibilité des candidats du KMT (trois en 2009 : à Miaoli, Da'an à Taipei et à Yunlin; deux en 2010 : dans les comtés de Taichung et de Taoyuan), et cinq autres pour remplacer des parlementaires élus comme maires ou chefs de comté en décembre 2009 (dans le comté de Chiayi, de Hsinchu, de Hualien, à Taitung et à Taoyuan). Sur ces dix élections, le DPP en a remporté sept, dont six organisées en janvier et en février 2010, pour un gain total de six sièges, confirmant ainsi ses bons résultats lors des élections régionales de décembre. Non seulement les candidats du DPP ont battu leurs opposants du KMT à Chiayi et à Yunlin, deux comtés favorables au camp vert, mais aussi lors des deux élections partielles à Taoyuan, ainsi que dans les comtés de Taichung, Hsinchu et Taitung, tous plus favorables au camp bleu, où ils ont remplacé des parlementaires du KMT. Dans le même temps les élections partielles de Miaoli ont été remportées par un candidat dissident du KMT soutenu par le DPP, ne laissant que deux tièdes victoires au KMT dans le district de Da'an à Taipei et à Hualien, bastions du camp bleu.

Par conséquent, la majorité du KMT au Yuan législatif a diminué de 81 à 74 sièges, tandis que le nombre de parlementaires du DPP a augmenté de 27 à 33 . Avec ses alliés indépendants, le KMT contrôle toujours jusqu'à 78 sièges ${ }^{(59)}$, une majorité très confortable qui lui permet de faire voter toutes les lois qu'il désire, dans la mesure où la mauvaise communication entre le gouvernement et le groupe parlementaire du KMT n'entrave pas l'approbation des politiques et budgets gouvernementaux, comme lors $\mathrm{du}$ fiasco de l'autorisation du bœuf américain et d'autres cas de désaccords entre les deux branches du gouvernement ces deux dernières années.

\section{La résurgence du DPP}

Parallèlement à la chute de la popularité de Ma, le paysage politique des deux dernières années a été caractérisé par une résurgence progressive du DPP attestée par ses récents succès électoraux. Elle est due aussi bien à la déception de l'électorat envers le KMT qu'au changement de direction et de discours du DPP. Depuis les écrasantes défaites de 2008, le DPP s'est engagé dans une double politique de réforme et de reconstruction fondée sur la consolidation de ses soutiens traditionnels et une expansion vers le segment crucial des électeurs flottants. Sous la nouvelle direction de Tsai Ing-wen, le parti s'est progressivement dissocié de Chen Shui-bian et des scandales de corruption entourant sa famille tout en continuant à défendre les réussites des huit années de gouvernement du DPP en termes de croissance économique, de promotion de la souveraineté et de la dignité de Taiwan, et de politiques sociales. Il a toutefois été attentif à ne pas donner l'impression d'abandonner l'ancien président qui recueille toujours la sympathie et le soutien des militants "verts foncés" qui considèrent les poursuites judiciaires engagées contre lui comme une forme de vendetta du KMT. Dans le même temps, la nouvelle direction tente de renforcer la capacité du parti de formuler des politiques centrées sur les besoins de la population, et particulièrement des classes moyennes et populaires, en développant son expertise et ses compétence techniques. Le parti promeut aussi les résultats des gouvernements de villes et comtés DPP afin de renforcer son image de parti proche de la population et aux bonnes pratiques de gouvernance. Cela fonctionne bien avec l'arrière-plan académique de Tsai Ing-wen et son image d'experte et a contribué à modérer l'image radicale trop souvent associée au DPP sous la présidence de Chen Shui-bian. Des affrontements occasionnels lors de manifestations publiques et des pugilats au Yuan législatif empêchent toujours la rénovation complète de l'image du parti. Mais ceux-ci témoignent aussi de l'intensité de la frustration et de la colère ressenties par les partisans du camp vert à propos de la direction donnée actuellement au pays et leur sentiment d'impuissance à en influencer l'évolution. Après sa série de succès électoraux, le DPP a retrouvé une certaine unité et confiance en soi. Les sondages d'opinion montrent aussi que depuis novembre 2009 Tsai Ing-wen connait une popularité

58. "A Travesty of Democracy ", China Post, 7 décembre 2009; Peter Harmsen, "Votebuying Taints Taiwan's Democracy: Analysts », Agence France-Presse, 2 décembre 2009.

59. En juillet 2010, un autre parlementaire du camp bleu et seul député du PFP (représentant les « aborigènes des Plaines ») a vu son élection annulée pour achat de voix. 
supérieure à Ma Ying-jeou ${ }^{(00)}$ et le parti continuera certainement à profiter des erreurs et des échecs du gouvernement et du KMT. Mais pour gagner des élections nationales à l'avenir, il lui faudra également se transformer en parti plus attentif aux problèmes de base de la population et proposer une politique claire et (peut-être plus) pragmatique concernant la relation de Taiwan avec la Chine et la question de l'indépendance afin d'obtenir un soutien plus large auprès de l'électorat du milieu.

Le prochain test électoral d'importance aura lieu le 27 novembre 2010 avec l'élection des maires et des conseils municipaux des cinq municipalités spéciales. Le DPP peut compter sur les réussites de ses édiles locales dans les anciens comtés et villes de Kaohsiung et de Tainan qui, en plus de son poids traditionnellement plus important dans le sud, lui donnent l'avantage dans ces deux nouvelles municipalités s'il parvient à éviter les divisions internes. La bataille pour les municipalités spéciales de Taipei, Sinbei et Taichung dans des régions plus favorables au camp bleu, dépendra de la qualité des candidats du DPP et du KMT, de l'unité interne des deux camps et du bilan général des gouvernements national et locaux du KMT ${ }^{(6)}$. Pour le $\mathrm{KMT}$, la victoire dans ces trois municipalités est essentielle pour prouver que son déclin est enrayé et se placer en bonne position pour les élections de 2012 .

Les élections législatives et présidentielles de 2012 sont encore loin et beaucoup de choses peuvent se produire d'ici là, particulièrement dans le climat politique volatile de Taiwan. Le changement principal de ces deux dernières années est néanmoins que la réélection de $\mathrm{Ma}$ Ying-jeou n'est plus assurée. Des sondages du printemps 2010 ont même montré que Ma pourrait perdre l'élection dans le cas d'une candidature de Su Tseng-chang ${ }^{(2)}$. En 2008, un second mandat était considéré comme acquis autant par le KMT que par l'opposition. Que cela ne soit plus le cas démontre à quel point la politique et les performances gouvernementales du KMT et de Ma Ying-jeou ont déçu la population. L'élection de 2012 reste à la portée de $\mathrm{Ma}$, tant il bénéficie des avantages du sortant ainsi que des réseaux, de l'argent et des médias du gouvernement et du KMT. Mais pour être réélu, il faudra que l'économie connaisse une nette amélioration et que son gouvernement soit plus à l'écoute des inquiétudes et des besoins de la population. Ma devra aussi peut-être avancer plus prudemment sur le plan des relations avec la Chine et de la souveraineté de Taiwan alors que le fossé se creuse entre son gouvernement et le reste de la population au sujet de l'identité nationale.

\section{Ma Ying-jeou et l'identité nationale de Taiwan}

Ma n'a pas été élu sur un programme de réunification avec la Chine ni sur un programme nationaliste chinois. Bien au contraire, il a mené une campagne autour de sa propre identité taiwanaise, de la défense de la souveraineté de Taiwan en tant que République de Chine, et de l'engagement que l'avenir de Taiwan serait décidé par les seuls 23 millions de Taiwanais ${ }^{(63)}$. Ce qui inquiète de nombreux Taiwanais, audelà des $41,5 \%$ qui ont voté contre lui, est la quasi-disparition, depuis son élection, de son discours taiwanais et son remplacement par un discours nationaliste chinois centré sur la République de Chine et la grande ethnie/nation chinoise (Zhonghua minzu). Une prise de conscience des convictions nationalistes chinoises de Ma s'est manifestée dans les sondages sur l'attitude du président envers la question de l'indépendance et de l'unification ${ }^{(64)}$. Alors qu'en août 2005, $46,9 \%$ des personnes interrogées pensaient que la position de Ma était la préservation du statu quo contre $24 \%$ qui estimaient qu'il favorisait l'unification, en octobre 2009 seuls $30,1 \%$ croyaient toujours que Ma était en faveur du statu quo sur le long terme contre $45,1 \%$ qui considéraient qu'il soutenait l'unification.

Un problème majeur de la rhétorique prochinoise de $\mathrm{Ma}$, mis à part le fait qu'elle s'écarte de son discours de campagne, est qu'elle va à l'encontre de l'évolution des Taiwanais sur la question de leur identité et de leur perception de la Chine. Les sondages d'opinion semblent même indiquer que plus le gouvernement du KMT se penche vers la Chine et s'accoquine avec les officiels de la RPC et plus les Taiwanais rencontrent de Chinois, plus la population s'identifie comme taiwanaise et différente des Chinois. Après le retour du KMT au pouvoir en 2008, certains à Taiwan et à l'étranger s'attendaient à ce que la progression du nationalisme et de l'identité nationale Taiwanaise s'arrête ou s'estompe.

60. Cf. Global Views Survey Research Center, «Taiwan Public Mood Index June 2010 », 25 juin 2010.

61. En juin 2010, tous les candidats étaient choisis. Ce sont : à Taipei, Hau Lung-bin (KMT) contre Su Tseng-chang (DPP) ; à Sinbei, Eric Chu Li-luan (KMT) contre Tsai Ing-wen (DPP) ; à Taichung, Jason Hu Chih-chiang (KMT) contre Su Jia-chyuan (DPP); à Tainan, Kuo Tien-tsai (KMT) contre William Lai Ching-te (DPP) et à Kaohsiung, Huang Chao-shun (KMT) contre Chen Chu (DPP).

62. «Public Opinion Poll. Survey Topic: 2012 Presidential Election », United Daily News, 19 mars 2010, http://udn.com/NEWS/NATIONAL/NAT1/5484850.shtml.

63. Cf. F. Muyard, «Élections 2008 à Taiwan », art. cit .; Daniel Lynch, « Mr. Ma’s Taiwanese Identity », Far Eastern Economic Review, vol. 171, n² 2, mars 2008.

64. Cf. Global Views Survey Research Center, « Survey on President Ma Ying-jeou's Performance after Assuming KMT Chairpersonship etc ». 22 octobre 2009, www.taiwansecurity.org/2009/GVMaApproval_Independence-102209.pdf. 
Cela était particulièrement vrai chez ceux qui pensaient que ces phénomènes étaient principalement dus aux manipulations politiques des gouvernements précédents de Lee Tenghui et de Chen Shui-bian. D'autres croyaient que sous un nouveau gouvernement prochinois, l'intégration économique croissante entre Taiwan et la Chine donnerait naissance à la montée d'une identité chinoise " commune " et à un désir d'unification politique chez les Taiwanais. Comme toutes les enquêtes le montrent depuis 2008, non seulement ce n'est pas le cas, mais l'identité taiwanaise et l'opposition à l'unification ont en fait continué à progresser, atteignant un pic historique sous la présidence de $\mathrm{Ma}$, en particulier chez les jeunes.

Selon les sondages du Centre d'enquêtes électorales de I'Université Chengchi (NCCU), la proportion de personnes interrogées qui se disaient taiwanaises en décembre 2007 était de $43,7 \%$ contre $44,5 \%$ qui se disaient à la fois taiwanais et chinois et $5,4 \%$ qui se déclaraient chinois ${ }^{(6)}$. En juin 2008 , les chiffres étaient respectivement de $46,1 \%$ contre $45,4 \%$ et $3,4 \%$. En juin 2010, 52,4 \% des personnes interrogées s'identifiaient comme taiwanaises - un bon de 6,3\% en deux ans -, contre $40,4 \%$ qui déclaraient une double identité et seulement $3,8 \%$ qui se considéraient chinois. La hausse continue du nombre de personnes se considérant taiwanaises par rapport à la fois à la double identité et à l'identité chinoise s'est donc poursuivie même après la victoire de Ma de 2008, faisant de l'identité taiwanaise le choix majoritaire depuis décembre 2008, et celui recueillant le plus haut score jamais atteint pour toute catégorie avec 52,4 \% en juin 2010. Les sondages du groupe de médias TVBS proche du camp bleu confirment cette tendance, et montrent que, lorsqu'on leur demandait en mars 2009 de choisir entre seulement deux options, $72 \%$ des personnes interrogées se déclaraient taiwanaises contre $16 \%$ qui se disaient chinoises, une augmentation de $4 \%$ et un déclin de $2 \%$ respectivement depuis juin $2008^{(66)}$. Un sondage plus récent et complet du CommonWealth Magazine indique également qu'en décembre 2009, $62 \%$ des personnes interrogées s'identifiaient comme taiwanaises, contre $22 \%$ à la fois taiwanaises et chinoises et $8 \%$ chinoises ${ }^{(67)}$. La tendance est encore plus accentuée chez les jeunes : $75 \%$ des $18-29$ ans se considèrent taiwanais, contre $14 \%$ taiwanais et chinois et seulement $7 \%$ chinois.

Sur la question de l'indépendance et de l'unification, les sondages montrent aussi une évolution claire contre l'unification et en faveur de la séparation si ce n'est de l'indépendance formelle de Taiwan. Si l'option du statu quo recueille toujours le plus haut score dans tous les sondages, mises en de- meure de choisir plus spécifiquement entre l'indépendance et l'unification, les personnes interrogées se montrent très majoritairement favorables à la première. Les enquêtes de TVBS indiquent qu'en décembre 2009, $68 \%$ des Taiwanais soutenaient l'indépendance contre $13 \%$ qui souhaitaient l'unification et $19 \%$ qui préféraient ne pas répondre. Ceci constitue une progression de $13 \%$ en faveur de l'indépendance et une chute de $12 \%$ pour l'unification depuis mars $2007^{(68)}$. Une autre série de sondages du Global Views Magazine indique qu'en juillet 2010, 66,1 \% des Taiwanais s'opposaient à l'unification avec la Chine même si les deux pays étaient à un niveau de développement politique et économique similaire contre $12,1 \%$ qui y seraient favorables ${ }^{(69)}$. Le rejet de l'unification ne se fonde donc pas seulement sur une préférence pour la liberté et la démocratie par rapport à la dictature. Il reflète aussi clairement la perception de l'existence de deux sociétés distinctes et du désir de maintenir le pays séparé de la Chine.

Globalement, les Taiwanais ont une attitude plutôt pragmatique et matérialiste envers la Chine. En 2009, 52,3 \% d'entre eux considéraient les " gens du continent " comme des partenaires commerciaux alors que $13,3 \%$ les tenaient pour des amis ${ }^{(0)}$. Interrogés sur leurs motivations pour un éventuel voyage en Chine, $73,2 \%$ indiquent le tourisme, suivis par le travail ou les affaires pour seulement $14,5 \%$ et 3,5 $\%$ pour rendre visite à de la famille ou des amis. Mais les Taiwanais ont également une opinion de plus en plus négative de la Chine et des Chinois. Selon un sondage CommonWealth de septembre 2009, ceci n'est pas dû à l'ignorance mais, au contraire, à des contacts répétés : plus les gens ont l'expérience de la Chine, plus ils expriment de scepticisme ou de peur à son égard ${ }^{(71)}$. Ce sondage indique également que $70 \%$ des Taiwanais ont une opinion négative des tou-

65. Cf. «Important Political Attitude Trend Distribution », juin 2010, Election Study Center, National Chengchi University, http://esc.nccu.edu.tw/english/modules/tinyd2/index. php?id=6.

66. Cf. Sondage d'opinion publique TVBS, 12 mars 2009.

67. Cf. « 2010 State of the Nation Survey », CommonWealth Magazine, $n^{\circ} 437,15$ décembre 2009. http://english.cw.com.tw/article.do?action=show\&id=11589.

68. Cf. Sondage d'opinion publique TVBS, 13 mars 2009, op. cit., et sondage d'opinion publique TVBS, 17 décembre 2009.

69. Cf. Global Views Survey Research Center, "Survey on Signed ECFA etc. ", art. cit.

70. De l'autre côté, $53,3 \%$ des Chinois interrogés considéraient les "gens de Taiwan » comme de la famille ou des parents et seulement 16,2 \% comme des partenaires commerciaux. Cf. Global Views Research Center, « Survey on how each side of Taiwan Strait views the other », 1er juillet 2009, www.kmt.org.w/english/page/aspx?type=article\& mnum=114\&anum $=6489$

71. Jerry Lai, «Survey on Cross-strait Relations. Three out of Four Taiwanese Have Sinophobia», CommonWealth Magazine, $\mathrm{n}^{\circ}$ 430, 10 septembre 2009, http://english.cw.com.tw/ article.do?action $=$ show\&id $=11340$. 
ristes chinois, tandis que $70 \%$ craignent que les échanges inter-détroit ne fassent du tort à la souveraineté de Taiwan, que $75 \%$ ont peur que des interactions plus étroites avec la Chine menacent leur emploi ou leurs moyens d'existence, et que $60 \%$ estiment que les nouvelles relations inter-détroit profitent principalement au KMT et au PCC. Cela ne signifie pas que les Taiwanais sont contre les relations avec la Chine mais ils sont divisés sur la question de leurs avantages ( $48 \%$ pensent qu' elles sont largement bénéfiques tandis que $43 \%$ pensent que les inconvénients dépassent les avantages), et s'inquiètent de ce que le rapprochement économique avec la Chine et la politique prochinoise actuelle se fasse contre leurs intérêts.

Toutes les enquêtes d'opinion depuis la victoire présidentielle de Ma Ying-jeou indiquent donc que le changement de gouvernement n'a pas inversé les tendances de fond de l'identité nationale taiwanaise. Contrairement à la période de la dictature du KMT, depuis les années 1990 les Taiwanais sont libres de choisir et d'exprimer leur sentiment d'appartenance nationale et se sont prononcés de plus en plus clairement pour maintenir séparée de la Chine la société et la nation taiwanaises inclusives qu'lls partagent, même dans le cas de conditions économiques et politiques comparables entre les deux pays. Depuis le tournant des années 1990, les évolutions de la société taiwanaise ont en effet suivi un chemin de plus en plus distinct de celui de la Chine. Alors que certains pensaient que les deux sociétés convergeraient grâce au commerce et au capitalisme, elles ont au contraire eu tendance à s'éloigner davantage l'une de l'autre avec la démocratisation et le remplacement générationnel de Taiwan. Bien sûr, le grand nombre de Taiwanais qui travaillent en Chine a généré des contacts accrus entre les populations des deux pays. Mais ceci ne les rapproche pas nécessairement des Chinois ni ne les conduit à partager la même communauté de vie. Cela a également entrainé une meilleure appréhension des différences entre les deux sociétés dans de nombreux domaines : modes de vie, éducation, habitudes comportementales, relations avec l'autre, ouverture d'esprit, appréciation des cultures et pays étrangers (particulièrement les États-Unis et le Japon), systèmes sociaux et de santé, état de droit, en plus de la liberté politique et de la démocratie. Aujourd'hui, les contacts accrus avec les Chinois, soit en RPC pour le commerce et le tourisme, soit à Taiwan avec le nombre croissant de touristes chinois, semblent générer le même sentiment de différence envers les Chinois que lorsque les voyages en Chine commencèrent à être permis à la fin des années 1980. La politique de Ma de rapprochement avec la Chine semble également engendrer chez les
Taiwanais un plus fort désir d'affirmer leur identité taiwanaise et leur souhait d'indépendance.

\section{Conclusion}

En terme d'identité nationale, Ma et la plupart des politiciens du KMT des vieilles générations, en particulier ceux d'origine continentale, se trouvent aujourd'hui en décalage et en déconnection profonde avec le reste de la population que ce soit les Taiwanais de souche que les Aborigènes ou les plus jeunes générations de Continentaux ${ }^{(72)}$. La perte de confiance des Taiwanais envers le gouvernement de $\mathrm{Ma}$ Ying-jeou et les problèmes quil rencontre résultent en partie de ce décalage. Il montre également que Ma et son gouvernement ne comprennent pas ou ne veulent pas accepter l'évolution de l'identité politique et nationale taiwanaise de ces deux dernières décennies. L'élection de Ma Ying-jeou à la présidence de Taiwan a montré que les Taiwanais souhaitaient profiter davantage de la croissance économique chinoise et avoir une relation aussi apaisée que possible avec la Chine, comme promis par Ma lors de sa campagne. Mais ils continuent à affirmer leur indépendance, leur souveraineté et leur identité nationale taiwanaise. La capacité de $\mathrm{Ma}$ et du KMT à reconnaître cette réalité et à commencer à représenter l'identité de la majorité de la population plutôt que de la nier sous un discours d'ethnicité chinoise sera un des facteurs en jeu lors de la prochaine élection. Avec la performance socio-économique du pays et la compétence administrative de son gouvernement, cela déterminera si Ma peut remporter un second mandat ou si le DPP aura une autre occasion de gouverner le pays. $\bullet$

\section{- Traduit par Céline Letemplé et l'équipe de rédaction}




\section{Glossaire}

Chen Chu 陳菊

Chen Shui-bian 陳水扁

Chen Yunlin 陳雲林

Chiang Pin-kun 江丙坤

Chiayi 嘉義

Da-an 大安

Iles Diaoyutai/Senkaku - 釣魚台群岛 (en chinois) /

尖閣諸岛 (en japonais)

duideng de diqu dui diqu teshu de guanxi 對等的地區数地區特 殊的關係

Eric Chu Li-luan 朱立倫

Frank Hsieh Chang-ting 謝長廷

guozu 國族

Hau Lung-bin 郝龍斌

Hsinchu 新竹

Hualien 花蓮

Huang Chao-shun 黄昭順

Jason Hu Chih-chiang 胡志強

Kaohsiung 高雄

Keelung 基隆

Kinmen 金門

Kuo Tien-tsai 郭添財

Kuomintang 國民黨

Lee Teng-hui 李登輝

Lien Chan 連戰

Liu Chao-hsuan 劉兆玄

Matsu馬祖

Ma Ying-jeou 馬英九

Miaoli 苗栗

minzu 民族 minzuxue 民族學

Penghu 澎湖

Pingtung 屏東

renminbi 人民幣

shaoshu minzu 少數民族

Sinbei 新北

Su Chi 蘇起

Su Jia-chyuan 蘇嘉全

Su Tseng-chang 蘇貞昌

Taichung 台中

Tainan 台南

Taipei 台北

Taishang 台商

Taitung 台東

Tangwai 黨外

Taoyuan 桃園

teshu guo yu guo guanxi 特殊國與國關係

Tsai Eng-meng 蔡衍明

Tsai Ing-wen 蔡英文

Vincent Siew (Hsiao Wan-chang) 蕭萬長

William Lai Ching-te 賴清德

Wu Den-yih 员敦義

Wu Po-hsiung 只伯雄

yi bian yi guo 一邊一國

yi ge Zhongguo, ge zi biaoshu一個中國, 各自表述

yi zhong ge biao 一中各表

Yunlin 雲林

Zhonghua minzu 中華民族

zuqun 族群 